\title{
Quantum Kramers' equation for energy diffusion and barrier crossing dynamics in the low friction regime
}

\author{
Dhruba Banerjee ${ }^{1}$, Suman Kumar Banik ${ }^{1 *}$, Bidhan Chandra Bag ${ }^{2}$ and Deb Shankar Ray ${ }^{1 \dagger}$ \\ ${ }^{1}$ Indian Association for the Cultivation of Science, Jadavpur, Calcutta 700 032, India \\ ${ }^{2}$ Department of Chemistry, Visva-Bharati, Shantiniketan 731 235, India
}

(Dated: August 7, 2002)

\begin{abstract}
Based on a true phase space probability distribution function and an ensemble averaging procedure we have recently developed [ Phys. Rev. E 65, 021109 (2002) ] a non-Markovian quantum Kramers' equation to derive the quantum rate coefficient for barrier crossing due to thermal activation and tunneling in the intermediate to strong friction regime. We complement and extend this approach to weak friction regime to derive quantum Kramers' equation in energy space and the rate of decay from a metastable well. The theory is valid for arbitrary temperature and noise correlation. We show that depending on the nature of the potential there may be a net reduction of the total quantum rate below its corresponding classical value which is in conformity with earlier observation. The method is independent of path integral approaches and takes care of quantum effects to all orders.
\end{abstract}

PACS number(s): 05.40.-a, 82.20.-w

\section{INTRODUCTION}

The dynamics of noise-induced rate processes was first successfully treated in a seminal paper by Kramers in 1940 [1]. With the advances in experimental methods for monitoring ultrafast processes on microscopic spatial and temporal scales over the last two decades [2, 3], this has been the subject of numerous investigation from classical, semiclassical and quantum mechanical point of view [4 [7]. The classical Kramers' theory has thus been extended to non-Markovian dissipation models [8 10], generalization to complex potential [11 13] and to many degrees of freedom [14, 15], fluctuating barrier problem [16 and non-stationary activated processes [17], thermal ratchet [18, 19$]$ and molecular motors [20], analysis of quantum [4,5, 21 [23] and semiclassical effects [24], calculation of time-dependent transmission coefficient [25].26], fractional kinetics [27, 28], nonequilibrium open systems [29,30], activationless escape of a free Brownian particle [31] and other related issues [4,6.7].

Although classical Kramers' equation was proposed more than sixty years ago and quantum Kramers' problem of escape from a metastable state has attracted wide attention over the last two decades [4], the quantum version of Kramers' equation was not reported in the literature. This is probably because of the fact that the traditional method of treatment of quantum Kramers' problem rests on calculation of partition function for a system-reservoir Hamiltonian in terms of path integrals, rather than on evolution of probability distribution function as used in classical theory of stochastic processes. Very recently we have developed [32] a method based on true quantum (c-number) phase space distribution function (rather than quasi-probability function, like Wigner function [33]) to derive for the first time an exact non-Markovian quantum Kramers' equation which is valid for arbitrary temperature and friction. The solution of this equation as an appropriate boundary value problem results in an expression for quantum rate coefficient which not only reduces to Kramers'-Grote-Hynes [1] 8] rate in the classical limit but also to the result corresponding to zero-temperature tunneling in the full quantum limit, treated by Caldeira and Leggett [34] in early eighties. The rate coefficient thus derived pertains to spatially-diffusion limited processes and is therefore valid for intermediate to strong friction regime. We undertake the present study with the following specific objectives to complement this work in the low friction regime where the process is controlled by energy diffusion.

(1) To extend the treatment of quantum Kramers' problem for low to low-moderate friction we develop a quantum Kramers' equation for energy diffusion which is a quantum version of classical non-Markovian equation of Carmeli and Nitzan $(\mathrm{CN})$ 10 proposed in early eighties.

(2) Our aim here is the inclusion of memory effects for arbitrary noise correlation of the heat bath kept at an arbitrary temperature taking into consideration of the quantum effects (corrections) to all orders.

\footnotetext{
*Present Address: Max-Planck-Institut für Physik komplexer Systeme, Nöthnitzer Straße 38, 01187 Dresden, Germany

${ }^{\dagger}$ Email address: pcdsr@mahendra.iacs.res.in
} 
(3) We solve the quantum Kramers' equation for energy diffusion to derive an explicit form of rate coefficient in the weak friction regime and show that it reduces to non-Markovian counterpart of Hänggi and Weiss [35] in the classical limit. Furthermore it provides the rate coefficient of low temperature tunneling (down to absolute zero) in the quantum limit. The present theory thus interpolates between thermal activation and tunneling for weak dissipation within a single scheme and is a direct extension of classical theory to quantum domain.

The classical non-Markovian Fokker-Planck equation in the energy variable for arbitrary noise correlation was first proposed by $\mathrm{CN}$ [10]. The detailed classical analysis by several groups 10,35 37] revealed that the rate, in general, is significantly modified by memory effects when compared to corresponding Kramers' theory in the static friction limit. As mentioned earlier, the traditional quantum treatment of the Kramers' problem in weak friction limit on the other hand, is based on functional integral approach [4] which takes care of dissipative tunneling [34]. Since for weak friction limit at a finite temperature one finds a small population at the upper energy levels of the system which results in non-equilibrium effects, quantum correction to classical Kramers' weak damping results above the cross-over temperature is of considerable interest. Several authors 38 43 have addressed this problem in relation to nonequilibrium quantum tunneling out of a metastable state. Although the method of functional integrals as employed in these theories has been successful in treating arbitrary coupling and correlation time scales in a formally exact manner, analytic evaluation of these integrals usually requires semi-classical approximations, e.g., semiclassical steepest descent method, WKB approach, etc., or other specific cases, which put restriction on applicability of the theories in several ways. Secondly, the weak coupling theories ( which have been extensively used in quantum optics [44. since seventies ) based on master equations that make use of quasi-probability functions, like Wigner function [33] often pose serious difficulties concerning negativity or singularity of the probability distribution functions as discussed in detail in earlier work [45]. Third, when the system or the system-reservoir coupling is nonlinear, the differential equations concern higher (than second) derivatives of quasi-probability functions [46] for which quantum-classical correspondence gets blurred. Our approach here is based on true quantum probability phase space function and is free from such difficulties. Furthermore an important decisive advantage of the scheme is that it allows us to implement the classical non-Markov theories of activated processes in a full quantum setting without taking recourse to any semiclassical technique. In what follows we specifically apply the classical procedure of Lax [47, CN [10], Büttiker, Harris and Landauer (BHL) [48], Hänggi and Weiss [35] in our quantum phase space formulation to develop a nonMarkovian quantum Kramers' equation in energy variable and derive an expression for quantum rate coefficient in the spirit of classical Kramers' theory. The quantum Kramers' equation and the rate coefficient are classical looking in form but quantum mechanical in their content and it is easy to recover their non-Markovian classical counterparts in the limit $\hbar \rightarrow 0$.

The Kramers' kinetics in the low friction regime is just not a theoretical issue today but has been a subject of experimental investigation over the last two decades [4,49, 53]. A number of experimental work in chemistry aimed at detecting Kramers' turnover phenomena, in various reactions which can be conveniently explained in terms of a one-dimensional model, e.g., iodine atom recombination in various inert solvents [49], chair-chair isomerization of cyclohexane [50], excited state isomerization of 2-alkylanthracene [52]. Another class of experiments where the energy diffusion mechanism has been successfully implemented concern resonant activation of a Josephson junction [37, 4 , and decay of zero voltage state in a current-biased Josephson junction [53]. The non-exponential decay behaviour in spin glass [- $[$ is also an area of active research in this context. All these problems have their quantum counterparts which are being considered for further studies in rate theory although the experimental evidence of some of the theoretical predictions in low temperature quantum effects in weak friction regime is still awaited.

The outlay of the paper is as follows. We introduce a $c$-number representation of a generalized quantum Langevin equation in Sec. II. This formulation helps us to use the classical formulation of CN [10] for deriving a non-Markovian quantum Kramers' equation in energy space in Sec. III. We solve the problem of quantum energy diffusion controlled rate coefficient in the spirit of classical theory [35, 48] in Sec. IV. This reduces to classical rate expression of KramersHänggi-Weiss 35 form in the limit $\hbar \rightarrow 0$. An explicit example with a cubic potential is worked out to illustrate the theory in Sec. V. The paper is concluded in Sec. VI.

\section{THE QUANTUM GENERALIZED LANGEVIN EQUATION (QGLE) IN $C$-NUMBERS}

We consider a particle in a medium. The latter is modeled as a set of harmonic oscillators with frequency $\left\{\omega_{i}\right\}$.

Evolution of such a quantum open system has been studied over the last several decades under a variety of reasonable assumptions. Specifically our interest here is to develop an exact description of quantum Brownian motion within the perview of this model described by the following Hamiltonian [54], 


$$
\hat{H}=\frac{\hat{P}^{2}}{2}+V(\hat{X})+\sum_{j}\left[\frac{\hat{p}_{j}^{2}}{2}+\frac{1}{2} \kappa_{j}\left(\hat{q}_{j}-\hat{X}\right)^{2}\right] .
$$

Here $\hat{X}$ and $\hat{P}$ are co-ordinate and momentum operators of the particle and the set $\left\{\hat{q}_{j}, \hat{p}_{j}\right\}$ is the set of co-ordinate and momentum operators for the reservoir oscillators coupled linearly to the system through their coupling coefficients $\kappa_{j}$. The potential $V(\hat{X})$ is due to the external force field for the Brownian particle. The co-ordinate and momentum operators follow the usual commutation relation $[\hat{X}, \hat{P}]=i \hbar$ and $\left[\hat{q}_{j}, \hat{p}_{j}\right]=i \hbar \delta_{i j}$. Note that in writing down the Hamiltonian no rotating wave approximation has been used.

Eliminating the reservoir degrees of freedom in the usual way [44,55,56] we obtain the operator Langevin equation for the particle,

$$
\ddot{\hat{X}}(t)+\int_{0}^{t} d t^{\prime} \beta\left(t-t^{\prime}\right) \dot{\hat{X}}\left(t^{\prime}\right)+V^{\prime}(\hat{X})=\hat{F}(t)
$$

where the noise operator $\hat{F}(t)$ and the memory kernel $\beta(t)$ are given by

$$
\hat{F}(t)=\sum_{j}\left[\left\{\hat{q}_{j}(0)-\hat{X}(0)\right\} \kappa_{j} \cos \omega_{j} t+\hat{p}_{j}(0) \kappa_{j}^{1 / 2} \sin \omega_{j} t\right]
$$

and

$$
\beta(t)=\sum_{j} \kappa_{j} \cos \omega_{j} t
$$

with $\kappa_{j}=\omega_{j}^{2}$ ( masses have been assumed to be unity ).

The Eq. (2.2) is an exact quantized operator Langevin equation which is now a standard textbook material 444] and for which the noise properties of $\hat{F}(t)$ can be defined using a suitable initial canonical distribution of the bath co-ordinates and momenta. Our aim here is to replace it by an equivalent QGLE in $c$-numbers. Again this is not a new problem so long as one is restricted to standard quasi-probabilistic methods using, for example, Wigner functions [33. To address the problem of quantum non-Markovian dynamics in terms of a true probabilistic description we, however, follow a different procedure. We first carry out the quantum mechanical average of Eq.(2.2)

$$
\langle\ddot{\hat{X}}(t)\rangle+\int_{0}^{t} d t^{\prime} \beta\left(t-t^{\prime}\right)\left\langle\dot{\hat{X}}\left(t^{\prime}\right)\right\rangle+\left\langle V^{\prime}(\hat{X})\right\rangle=\langle\hat{F}(t)\rangle
$$

where the average $\langle\ldots\rangle$ is taken over the initial product separable quantum states of the particle and the bath oscillators at $t=0,|\phi\rangle\left\{\left|\alpha_{1}\right\rangle\left|\alpha_{2}\right\rangle \ldots\left|\alpha_{N}\right\rangle\right\}$. Here $|\phi\rangle$ denotes any arbitrary initial state of the particle and $\left|\alpha_{i}\right\rangle$ corresponds to the initial coherent state of the $i$-th bath oscillator. $\left|\alpha_{i}\right\rangle$ is given by $\left|\alpha_{i}\right\rangle=\exp \left(-\left|\alpha_{i}\right|^{2} / 2\right) \sum_{n_{i}=0}^{\infty}\left(\alpha_{i}^{n_{i}} / \sqrt{n_{i} !}\right)\left|n_{i}\right\rangle$, $\alpha_{i}$ being expressed in terms of the mean values of the co-ordinate and momentum of the $i$-th oscillator, $\left\langle\hat{q}_{i}(0)\right\rangle=$ $\left(\sqrt{\hbar} / 2 \omega_{i}\right)\left(\alpha_{i}+\alpha_{i}^{\star}\right)$ and $\left\langle\hat{p}_{i}(0)\right\rangle=i \sqrt{\hbar \omega_{i} / 2}\left(\alpha_{i}^{\star}-\alpha_{i}\right)$, respectively. It is important to note that $\langle\hat{F}(t)\rangle$ of Eq. (2.5) is a classical-like noise term which, in general, is a non-zero number because of the quantum mechanical averaging over the co-ordinate and momentum operators of the bath oscillators with respect to the initial coherent states and arbitrary initial state of the particle and is given by

$$
\langle\hat{F}(t)\rangle=\sum_{j}\left[\left\{\left\langle\hat{q}_{j}(0)\right\rangle-\langle\hat{X}(0)\rangle\right\} \kappa_{j} \cos \omega_{j} t+\left\langle\hat{p}_{j}(0)\right\rangle \kappa_{j}^{1 / 2} \sin \omega_{j} t\right] .
$$

It is convenient to rewrite the $c$-number equation (2.5) as follows;

$$
\langle\ddot{\hat{X}}(t)\rangle+\int_{0}^{t} d t^{\prime} \beta\left(t-t^{\prime}\right)\left\langle\dot{\hat{X}}\left(t^{\prime}\right)\right\rangle+\left\langle V^{\prime}(\hat{X})\right\rangle=F(t)
$$

where we let the quantum mechanical mean value $\langle\hat{F}(t)\rangle=F(t)$. We now turn to the second average. To realize $F(t)$ as an effective $c$-number noise we now assume that the momenta $\left\langle\hat{p}_{j}(0)\right\rangle$ and the shifted co-ordinates $\left\{\left\langle\hat{q}_{j}(0)\right\rangle-\langle\hat{X}(0)\rangle\right\}$ of the bath oscillators are distributed according to a canonical distribution of Gaussian forms as

$$
\mathcal{P}_{j}=\mathcal{N} \exp \left\{\frac{-\left[\left\langle\hat{p}_{j}(0)\right\rangle^{2}+\kappa_{j}\left\{\left\langle\hat{q}_{j}(0)\right\rangle-\langle\hat{X}(0)\rangle\right\}^{2}\right]}{2 \hbar \omega_{j}\left(\bar{n}_{j}+\frac{1}{2}\right)}\right\}
$$


so that for any quantum mechanical mean value $O_{j}\left(\left\langle\hat{p}_{j}(0)\right\rangle,\left\{\left\langle\hat{q}_{j}(0)\right\rangle-\langle\hat{X}(0)\rangle\right\}\right)$ the statistical average $\langle\ldots\rangle_{S}$ is

$$
\begin{aligned}
\left\langle O_{j}\right\rangle_{S}= & \int O_{j}\left(\left\langle\hat{p}_{j}(0)\right\rangle,\left\{\left\langle\hat{q}_{j}(0)\right\rangle-\langle\hat{X}(0)\rangle\right\}\right) \\
& \times \mathcal{P}_{j}\left(\left\langle\hat{p}_{j}(0)\right\rangle,\left\{\left\langle\hat{q}_{j}(0)\right\rangle-\langle\hat{X}(0)\rangle\right\}\right) \\
& \times d\left\langle\hat{p}_{j}(0)\right\rangle d\left\{\left\langle\hat{q}_{j}(0)\right\rangle-\langle\hat{X}(0)\rangle\right\} .
\end{aligned}
$$

Here $\bar{n}_{j}$ indicates the average thermal photon number of the $j$-th oscillator at temperature $T$ and $\bar{n}_{j}=$ $1 /\left[\exp \left(\hbar \omega_{j} / k_{B} T\right)-1\right]$ and $\mathcal{N}$ is the normalization constant.

The distribution (2.8) and the definition of statistical average (2.9) imply that $F(t)$ must satisfy

$$
\langle F(t)\rangle_{S}=0
$$

and

$$
\left\langle F(t) F\left(t^{\prime}\right)\right\rangle_{S}=\frac{1}{2} \sum_{j} \kappa_{j} \hbar \omega_{j}\left(\operatorname{coth} \frac{\hbar \omega_{j}}{2 k_{B} T}\right) \cos \omega_{j}\left(t-t^{\prime}\right)
$$

That is, the $c$-number noise $F(t)$ is such that it is zero centered and satisfies the standard quantum fluctuationdissipation relation (FDR) as known in the literature [55] in terms of quantum statistical average of the noise operators. The distribution (2.8) is thus an ansatz introduced to calculate the ensemble average over the quantum-mechanical mean values of the bath oscillators. Its justification lies in the fact that with (2.9) it reproduces the correct noise properties of the bath, i.e., the quantum fluctuation-dissipation relation for the $c$-number quantum noise (2.11) along with (2.10). Secondly, the distribution (2.8) has a form which is Boltzmann-like (but not a Boltzmann distribution) since the width parameter of the Boltzmann distribution $k T$ gets replaced by $\hbar \omega_{j}\left(\bar{n}_{j}+\frac{1}{2}\right)$

To proceed further we now add the force term $V^{\prime}(\langle\hat{x}\rangle)$ on both sides of Eq.(2.7) and rearrange it to obtain formally

$$
\ddot{x}(t)+\int_{0}^{t} d t^{\prime} \beta\left(t-t^{\prime}\right) \dot{x}\left(t^{\prime}\right)+V^{\prime}(x)=F(t)+Q(x, t)
$$

where we let $\langle\hat{X}(t)\rangle=x(t)$ for simple notational convenience and

$$
Q(x, t)=V^{\prime}(x)-\left\langle V^{\prime}(\hat{X})\right\rangle
$$

represents the quantum mechanical dispersion of the force operator $V^{\prime}(\hat{X})$ due to the system degree of freedom. Since $Q(x, t)$ is a quantum fluctuation term Eq.(2.12) offers a simple interpretation. This implies that the classical looking QGLE is governed by a $c$-number quantum noise $F(t)$ which originates from the quantum mechanical heat bath characterized by the properties (2.10) and (2.11) and a quantum fluctuation term $Q(x, t)$ due to the quantum nature of the system characteristic of the nonlinearity of the potential. Although because of the last term in Eq.(2.12) the equation looks formal and implicit, the actual structure of $Q(x, t)$ gets more transparent as we go over to the beginning of the next section. A recipe for calculation of $Q(x, t)$ is given in Ref. [45,57,58].

We summarize the above discussions to point out that it is possible to formulate an exact QGLE (2.12) of the quantum mechanical mean value of position of a particle in a medium, provided the classical-like noise term $F(t)$ satisfies (2.10) and (2.11). The important new content of the approach is that to realize $F(t)$ as a noise term we have split up the standard quantum statistical averaging procedure into a quantum mechanical mean $\langle\ldots\rangle$ by explicitly using an initial coherent state representation of the bath oscillators and then a statistical average $\langle\ldots\rangle_{S}$ of the quantum mechanical mean values with distribution (2.8). This is distinctly different from the usual procedure of quantum statistical averaging where the quantum mechanical average is carried out with number states over the noise operators followed by an ensemble average with Boltzmann distribution. Two pertinent points are to be noted: First, it may be easily verified that the distribution of quantum mechanical mean values of the bath oscillators (2.8) reduces to classical Maxwell-Boltzmann distribution in the thermal limit, $\hbar \omega_{j} \ll k_{B} T$. Second, the vacuum term in the distribution (2.8) prevents the distribution of quantum mechanical mean values from being singular at $T=0$; or in other words the width of distribution remains finite even at absolute zero, which is a simple consequence of uncertainty principle. The procedure has been recently implemented by us to formulate a quantum theory of Brownian motion [45] and to propose an exact non-Markovian quantum Kramers' equation [32] with true probability distribution functions. 


\section{QUANTUM KRAMERS' EQUATION IN ENERGY SPACE}

Let us begin by noting that the generalized quantum Langevin equation (2.12) of a Brownian particle in presence of an external force field takes into account of arbitrary coupling between the system and heat bath and contains quantum corrections, $Q(x, t)$ due to system to all orders. To make the later assertion explicit we now express the operators $\hat{X}$ and $\hat{P}$ as

$$
\begin{aligned}
\hat{X}(t) & =\langle\hat{X}(t)\rangle+\delta \hat{X}(t) \\
\hat{P}(t) & =\langle\hat{P}(t)\rangle+\delta \hat{P}(t) .
\end{aligned}
$$

By construction $\langle\delta \hat{X}(t)\rangle=0,\langle\delta \hat{P}(t)\rangle=0$ and $[\delta \hat{X}, \delta \hat{P}]=i \hbar$. Expanding $\left\langle V^{\prime}(\hat{X})\right\rangle$ around $\langle\hat{X}\rangle(\equiv x)$ in a Taylor series we obtain

$$
\left\langle V^{\prime}(\hat{X})\right\rangle=V^{\prime}(x)+\frac{1}{2} V^{\prime \prime \prime}(x)\left\langle\delta \hat{X}^{2}\right\rangle+\ldots
$$

Therefore $Q(t)$ can be expressed as

$$
Q(t)=-\sum_{n=3}^{\infty} \frac{1}{(n-1) !} V^{n}(x)\left\langle\delta \hat{X}^{(n-1)}(t)\right\rangle
$$

Here $V^{n}(x)$ denotes the $\mathrm{n}$-th derivative of the classical potential. The role of $Q(x, t)$ is therefore to modify the classical potential $V(x)$ in Eq.(2.12). $Q(x, t)$ can be calculated by solving $\left\langle\delta \hat{X}^{n}(t)\right\rangle$ order by order. To the lowest order (second) $\langle\hat{X}\rangle$ and $\left\langle\delta \hat{X}^{2}\right\rangle$ follow a coupled set of equations as given in Eqs.55(a-e) of Ref. [45]. (Higher order equations, e.g., the fourth order equations, are given in Ref. [58]). For convenience, we will now split up the right hand side of Eq.(3.10) into a time-independent and a time-dependent part as,

$$
Q(t)=-\sum_{n=3}^{\infty} \frac{1}{(n-1) !} V^{n}(x)\left\langle\delta \hat{X}^{(n-1)}(0)\right\rangle+g(t)
$$

where

$$
g(t)=-\sum_{n=3}^{\infty} \frac{1}{(n-1) !} V^{n}(x)\left[\left\langle\delta \hat{X}^{(n-1)}(t)\right\rangle-\left\langle\delta \hat{X}^{(n-1)}(0)\right\rangle\right] .
$$

For future use it is convenient to write $g(t)$ in the Taylor series of the form,

$$
g(t)=-\sum_{m=0}^{\infty} \sum_{n=3}^{\infty} \frac{1}{(n-1) !} \frac{t^{m}}{m !}\left[\frac{\partial^{m}}{\partial t^{m}} V^{n}[x(t)]\left\{\left\langle\delta \hat{X}^{(n-1)}(t)\right\rangle-\left\langle\delta \hat{X}^{(n-1)}(0)\right\rangle\right\}\right]_{t=0} .
$$

The Langevin equation (2.12) then reduces to

$$
\ddot{x}+\int_{0}^{t} d \tau \beta(t-\tau) \dot{x}(\tau)+V^{\prime}(x)+\sum_{n=3}^{\infty} \frac{1}{(n-1) !} V^{n}(x)\left\langle\delta \hat{X}^{(n-1)}(0)\right\rangle=F(t)+g(t) .
$$

Expressing

$$
V_{q}(x)=V(x)+\sum_{n=3}^{\infty} \frac{1}{(n-1) !} V^{n-1}(x)\left\langle\delta \hat{X}^{(n-1)}(0)\right\rangle
$$

Eq.(3.2) takes the form

$$
\begin{gathered}
\dot{x}=v \\
\dot{v}+\int_{0}^{t} d \tau \beta(t-\tau) v(\tau)+V_{q}^{\prime}(x)=F(t)+g(t)
\end{gathered}
$$


Eq.(3.5a) is our starting Langevin equation. The potential $V_{q}(x)$ appearing in (3.3) and (3.5a) is not the classical potential but a renormalized one with quantum corrections. The damping memory kernel (2.4) is identified by the fluctuation-dissipation relation (2.11) by noting that in the continuum limit

$$
\langle F(t) F(0)\rangle_{S}=\frac{1}{2} \int_{0}^{\infty} d \omega \kappa(\omega) \rho(\omega) \hbar \omega\left(\operatorname{coth} \frac{\hbar \omega}{2 k_{B} T}\right) \cos \omega t \equiv C(t)
$$

and

$$
\beta(t)=\int_{0}^{\infty} d \omega \kappa(\omega) \rho(\omega) \cos \omega t
$$

in the Fourier domain can be related as

$$
\tilde{C}^{c}(\omega)=\frac{1}{2} \hbar \omega\left(\operatorname{coth} \frac{\hbar \omega}{2 k_{B} T}\right) \tilde{\beta}^{c}(\omega)
$$

where $\tilde{C}^{c}(\omega)$ and $\tilde{\beta}^{c}(\omega)$ are the cosine transforms of $C(t)$ and $\beta(t)$, respectively. For convenience we now express the Fourier transform of $\beta(t)$ as

$$
\tilde{\beta}_{n}(\omega)=\int_{0}^{\infty} d t \beta(t) \exp (-i n \omega t)
$$

We now consider the following time scales in the dynamics relevant for energy diffusion in the weak friction limit,

$$
\gamma \ll 1 / \tau_{c} \ll \omega
$$

where $\gamma$ is the friction arising due to interaction with the bath, evaluated in the Markovian limit. $\tau_{c}$ is the correlation time of the noise due to heat bath and $\omega$ is the linearized system frequency, which for a Brownian particle is assumed to be very high. This separation of time scales in (3.7) and casting of an operator Langevin equation in $c$-number form (3.4 3.5a) allow us to implement a classical method for solving the problem of quantum energy diffusion. Following the standard procedure one can transform Eqs. 3.4 3.5a) to the action $(\mathrm{J})$ and angle $(\phi)$ co-ordinates with the help of a Jacobian matrix as

$$
\begin{aligned}
\left(\begin{array}{l}
\dot{j} \\
\dot{\phi}
\end{array}\right) & =\left(\begin{array}{cc}
-\frac{\partial v}{\partial \phi} & \frac{\partial x}{\partial \phi} \\
\frac{\partial v}{\partial J} & -\frac{\partial x}{\partial J}
\end{array}\right)\left(\begin{array}{c}
\dot{x} \\
\dot{v}
\end{array}\right) \\
& =\left(\begin{array}{cc}
-\frac{\partial v}{\partial \phi} & \frac{\partial x}{\partial \phi} \\
\frac{\partial v}{\partial J} & -\frac{\partial x}{\partial J}
\end{array}\right)\left(\begin{array}{c}
\frac{\partial H}{\partial v} \\
-\frac{\partial H}{\partial x}-\int_{0}^{t} d \tau \beta(t-\tau) v(\tau)+F(t)+g(t)
\end{array}\right) .
\end{aligned}
$$

Thus we have

$$
\begin{aligned}
& \dot{J}=\frac{\partial x}{\partial \phi}\left[-\int_{0}^{t} d \tau \beta(t-\tau) v(\tau)+F(t)+g(t)\right] \\
& \dot{\phi}=\omega(J)-\frac{\partial x}{\partial J}\left[-\int_{0}^{t} d \tau \beta(t-\tau) v(\tau)+F(t)+g(t)\right]
\end{aligned}
$$

Here $v$ represents the velocity of the particle. For the deterministic part of the system's Hamiltonian given by $H=(1 / 2) v^{2}+V_{q}(x)$ we may write,

$$
\omega(J)=\frac{d H(J)}{d J} .
$$

Since $V_{q}(x)$ (see Eq.(3.3) ) contains quantum corrections, our $J$ and $\phi$ are quantum (c-number) variables as implied in (3.3). In the absence of quantum corrections they become classical variables of $\mathrm{CN}$ [10]. The canonical transformation from $(x, v)$ space to $(J, \phi)$ space has been done with the deterministic Hamiltonian. We can therefore expand $x$ and $v$ as,

$$
\begin{aligned}
& x(J, \phi)=\sum_{n=-\infty}^{\infty} x_{n}(J) \exp (i n \phi) \\
& v(J, \phi)=\sum_{n=-\infty}^{\infty} v_{n}(J) \exp (i n \phi)
\end{aligned}
$$


with

$$
x_{n}=x_{-n}^{*} \text { and } v_{n}=v_{-n}^{*} .
$$

Differentiating Eq. 3.11 a $)$ with respect to time and noting that in the action-angle variable space $\dot{\phi}=\omega(J)$ we can write,

$$
v_{n}(J)=i n \omega(J) x_{n}(J)
$$

Since, we are considering the motion in one dimension only, we can choose $J$ and $\phi$ in such a way that we can make the simplification for $x=x^{\star}$ as,

$$
x=\frac{1}{2} \sum_{n=-\infty}^{\infty}\left[x_{n} \exp (i n \phi)+x_{n}^{*} \exp (-i n \phi)\right]
$$

Inserting Eq.(3.12) we get,

$$
x=\frac{1}{2} \sum_{n=-\infty}^{\infty}\left[x_{n} \exp (i n \phi)+x_{-n} \exp (-i n \phi)\right] .
$$

With the choice of phase

$$
x=x_{-n} \quad\left[\operatorname{since} \operatorname{Im}\left(\mathrm{x}_{\mathrm{n}}\right)=0\right]
$$

$x$ may be further expressed as,

$$
x=\sum_{n=-\infty}^{\infty} x_{n} \cos n \phi
$$

Similarly using Eq. 3.13) and (3.14a) we get for $v_{n}=-v_{-n}$

$$
v=\sum_{n=-\infty}^{\infty} v_{n} \sin n \phi .
$$

Inserting Eq.(3.11a) and (3.11b) in Eq. (3.8) and (3.9) we obtain,

$$
\begin{aligned}
\dot{J}= & -i \sum_{n=-\infty}^{\infty} \sum_{m=-\infty}^{\infty} n x_{n} \exp (i n \phi) \int_{0}^{t} d \tau \beta(t-\tau) v_{m} \exp (i m \phi) \\
& +i S(t) \sum_{n=-\infty}^{\infty} n x_{n} \exp (i n \phi) \\
\dot{\phi}= & \omega(J)+\sum_{n=-\infty}^{\infty} \sum_{m=-\infty}^{\infty} \frac{\partial x_{n}}{\partial J} \exp (i n \phi) \int_{0}^{t} d \tau \beta(t-\tau) v_{m} \exp (i m \phi) \\
& -S(t) \sum_{n=-\infty}^{\infty} \frac{\partial x_{n}}{\partial J} \exp (i n \phi)
\end{aligned}
$$

where, we have expressed $S(t)$ as a sum of two terms; the noise due to heat bath, $F(t)$ and quantum correction term, $g(t)$

$$
S(t)=F(t)+g(t)
$$

In the equations of motion (3.15) and (3.16), the argument of the damping memory kernel $\beta$ is $(t-\tau)$. Now $\beta$ decays to zero in a time $\tau_{c}$ (the correlation time). So, to deal with the integrals of Eq.(3.15) and Eq.(3.16), it is reasonable to divide the range of integration into two parts: (a) $|t-\tau| \leq \tau_{c}$ and (b) $t \gg \tau_{c}$. Thus following $\mathrm{CN}$ [10] we can write 


$$
\begin{aligned}
\phi(t) & =\phi[\tau+(t-\tau)] \\
& \simeq \phi(\tau)+\left.\frac{\partial \phi}{\partial t}\right|_{t=\tau}(t-\tau),
\end{aligned}
$$

neglecting higher terms of $\tau_{c}$. It follows that,

$$
\begin{aligned}
\phi(\tau) & \simeq \phi(t)-(t-\tau) \omega \\
\text { and } \quad v_{m}(\tau) & \simeq v_{m}(t)
\end{aligned}
$$

Eq.(3.18) and Eq.(3.19) are reasonable approximations so far as the integrals of Eq.(3.15) and Eq.(3.16) are concerned. Within the integral, we therefore manipulate the behaviour of $\phi$ and $v_{m}$ for a time upto which $\beta(t-\tau)$ exists and also for the observational time at which $\beta$ has decayed to zero. So, more specifically we can write for $|t-\tau| \leq \tau_{c}$,

$$
\int_{0}^{t} d \tau \beta(t-\tau) v_{m}(\tau) \exp [i m \phi(\tau)] \simeq v_{m}(t) \exp [i m \phi(t)] \int_{0}^{t} d \tau \beta(t-\tau) \exp [-i m(t-\tau) \omega]
$$

and for $t \gg \tau_{c}$, using (3.6) we have

$$
\int_{0}^{t} d \tau \beta(t-\tau) v_{m}(\tau) \exp [i m \phi(\tau)] \simeq v_{m}(t) \exp [i m \phi(t)] \tilde{\beta}_{m}(\omega) .
$$

Putting Eq.3.21) which takes into account the observational time scale, in Eq.(3.15) and Eq.(3.16) we get,

$$
\begin{aligned}
\dot{J} & =-i \sum_{n=-\infty}^{\infty} \sum_{m=-\infty}^{\infty} n x_{n} v_{m} \tilde{\beta}_{m}(\omega) \exp [i(n+m) \phi]+i S(t) \sum_{n=-\infty}^{\infty} n x_{n} \exp (i n \phi) \\
\text { and } \dot{\phi} & =\omega(J)+\sum_{n=-\infty}^{\infty} \sum_{m=-\infty}^{\infty} x_{n}^{\prime} v_{m} \tilde{\beta}_{m}(\omega) \exp [i(n+m) \phi]-S(t) \sum_{n=-\infty}^{\infty} x_{n}^{\prime} \exp (i n \phi)
\end{aligned}
$$

where $x_{n}^{\prime}=\frac{\partial x_{n}}{\partial J}$.

Our next task is to formulate the Fokker-Planck equation. To this end we note that Lax 47 had prescribed a method for deriving Markovian Fokker-Planck equation from a classical Langevin equation with short but finite correlation time. Although the procedure can be extended to higher order iteration scheme to include non-Markovian effects we adopt the method advocated by Carmeli and Nitzan [10] for their classical theory. This is based on Kramers-Moyal expansion of the transition probability which connects the probability distribution function $P(J, \phi, t)$ at time $t$ with that of $P(J, \phi, t+\tau)$ at a later time $t+\tau$ for small $\tau$, given that we know the moments of the distribution. For details we refer to Risken [59] The time evolution of the probability distribution $P(J, \phi, t)$ is determined by the equation,

$$
\frac{\partial P}{\partial t}=\lim _{\tau \rightarrow 0+}\left[\frac{1}{\tau} \sum_{n=1}^{\infty} \frac{(-1)^{n}}{n !} \sum_{(m, k=0) ;(m+k=n)}\left(\frac{\partial}{\partial J}\right)^{m}\left(\frac{\partial}{\partial \phi}\right)^{k}\left\{\left\langle\left(\Delta J_{t}\right)^{m}\left(\Delta \phi_{t}\right)^{k}\right\rangle_{S} P\right\}\right]
$$

where

$$
\begin{aligned}
\Delta J_{t} & =\Delta J_{t}(\tau)=J(t+\tau)-J(t) \\
\Delta \phi_{t} & =\Delta \phi_{t}(\tau)=\phi(t+\tau)-\phi(t) .
\end{aligned}
$$

At this juncture it is worth recalling that $\tau$ is the coarse-grained time scale over which the probability distribution function evolves, whereas $\tau_{c}$ is the correlation time, which due to low damping is much smaller than $\tau$. The low value of $\gamma$ prompts us to take $\gamma^{-1}$ as the largest time scale for the entire problem. On the other hand, the reciprocal of the frequency of oscillation, i.e., $\omega^{-1}$, is the smallest time scale. Our task is therefore to evaluate the moments of the form $\left\langle\left(\Delta J_{t}\right)^{m}\left(\Delta \phi_{t}\right)^{k}\right\rangle_{S}$ where our definition of average $\langle\ldots\rangle_{S}$ is given in (2.9).

To evaluate the moments we make use of the following standard procedure [10,47]:

$$
\begin{aligned}
& \Delta J_{t}(\tau)=\int_{0}^{\tau} d s \dot{J}[J(t+s), \phi(t+s), t+s] \\
& \Delta \phi_{t}(\tau)=\int_{0}^{\tau} d s \dot{\phi}[J(t+s), \phi(t+s),(t+s)]
\end{aligned}
$$


where the forms $\dot{J}$ and $\dot{\phi}$ are given by Eq.(3.22) and (3.23), respectively. The iterative equations are given by,

$$
\begin{aligned}
& \Delta J_{t}^{(l)}(\tau)=\int_{0}^{\tau} d s \dot{J}\left[J(t)+\Delta J_{t}^{(l-1)}(s), \phi(t)+\Delta \phi_{t}^{(l-1)}(s), t+s\right] \\
& \Delta \phi_{t}^{(l)}(\tau)=\int_{0}^{\tau} d s \dot{\phi}\left[J(t)+\Delta J_{t}^{(l-1)}(s), \phi(t)+\Delta \phi_{t}^{(l-1)}(s), t+s\right]
\end{aligned}
$$

where $(l)$ denotes the $l^{\text {th }}$ iteration stage.

The non-Markovian nature (i.e., $\tau_{c}$ is finite and $\tau_{c}<\tau$ ) of the present problem allows us to consider, in principle, all orders of $\tau$ in Eq.(3.25). But, since $\partial P / \partial t$ is evaluated in the limit $\tau \rightarrow 0_{+}$, terms linear in $\tau$, i.e., the coarse-grained time scale, are taken while all the higher powers are neglected. We now introduce the following abbreviations

$$
\begin{aligned}
\sigma_{n}(J) & =i n x_{n}(J) \\
\mu_{n}(J) & =\frac{d x_{n}(J)}{d J} \\
B_{n m}(J) & =i n x_{n}(J) v_{m}(J) \tilde{\beta}_{m}[\omega(J)] \\
C_{n m}(J) & =\left[\frac{d x_{n}(J)}{d J}\right] v_{m}(J) \tilde{\beta}_{m}[\omega(J)]
\end{aligned}
$$

Substituting Eq.(3.30)-(3.33) in Eq.(3.22) and (3.23) we obtain the quantum equations in the form of classical equations of $\mathrm{CN}$ [10]:

$$
\begin{aligned}
\dot{J} & =-\sum_{n=-\infty}^{\infty} \sum_{m=-\infty}^{\infty} B_{n m}(J) \exp [i(n+m) \phi]+S(t) \sum_{n=-\infty}^{\infty} \sigma_{n}(J) \exp (i n \phi) \\
\text { and } \quad \dot{\phi} & =\omega(J)+\sum_{n=-\infty}^{\infty} \sum_{m=-\infty}^{\infty} C_{n m}(J) \exp [i(n+m) \phi]-S(t) \sum_{n=-\infty}^{\infty} \mu_{n}(J) \exp (i n \phi)
\end{aligned}
$$

From Eq.(3.28) and (3.34) we get the explicit structure of $\Delta J_{t}$ as,

$$
\begin{aligned}
\Delta J_{t}(\tau)= & -\sum_{n=-\infty}^{\infty} \sum_{m=-\infty}^{\infty} \int_{0}^{\tau} d s B_{n m}\left[J(t)+\Delta J_{t}(s)\right] \exp \left\{i(n+m)\left[\phi(t)+\Delta \phi_{t}(s)\right]\right\} \\
& +\sum_{n=-\infty}^{\infty} \int_{0}^{\tau} d s S(s) \sigma_{n}\left[J(t)+\Delta J_{t}(s)\right] \exp \left\{i n\left[\phi(t)+\Delta \phi_{t}(s)\right]\right\}
\end{aligned}
$$

Similarly from Eq.(3.29) and (3.35), $\Delta \phi_{t}$ is given by,

$$
\begin{aligned}
\Delta \phi_{t}(\tau) & =\int_{0}^{\tau} d s \omega\left[J(t)+\Delta J_{t}(s)\right] \\
& +\sum_{n=-\infty}^{\infty} \sum_{m=-\infty}^{\infty} \int_{0}^{\tau} d s C_{n m}\left[J(t)+\Delta J_{t}(s)\right] \exp \left\{i(n+m)\left[\phi(t)+\Delta \phi_{t}(s)\right]\right\} \\
& -\sum_{n=-\infty}^{\infty} \int_{0}^{\tau} d s S(s) \mu_{n}\left[J(t)+\Delta J_{t}(s)\right] \exp \left\{i n\left[\phi(t)+\Delta \phi_{t}(s)\right]\right\}
\end{aligned}
$$

For beginning the systematic iteration procedure given by Eq.(3.36) and (3.37) we initialize the zero order iteration stage as,

$$
\begin{aligned}
\Delta J_{t}^{(0)}(\tau) & =0 \\
\text { and } \quad \Delta \phi_{t}^{(0)}(\tau) & =\omega[J(t)] \tau=\omega \tau .
\end{aligned}
$$

The entire process of iteration involves cumbersome but straightforward calculations, some relevant details of which appear in the Appendix-A. Here we state only the main results.

Inserting Eqs.(3.38a and (3.38b) in the right hand side of Eqs. 3.36$)$ and (3.37) we get the results of the first order iteration. Thus, 


$$
\begin{aligned}
\Delta J_{t}^{(1)}(\tau) & =-\tau \sum_{n=-\infty}^{\infty} B_{n,-n}+\sum_{n=-\infty}^{\infty} \sigma_{n} \exp (i n \phi) \int_{0}^{\tau} d s F(s) \exp (i n \omega s) \\
& +\sum_{n=-\infty}^{\infty} \sigma_{n} \exp (i n \phi) \int_{0}^{\tau} d s g(s) \exp (\text { in } \omega s)
\end{aligned}
$$

and

$$
\begin{aligned}
\Delta \phi_{t}^{(1)}(\tau) & =\omega \tau+\tau \sum_{n=-\infty}^{\infty} C_{n,-n}-\sum_{n=-\infty}^{\infty} \mu_{n} \exp (i n \phi) \int_{0}^{\tau} d s F(s) \exp (\text { in } \omega s) \\
& -\sum_{n=-\infty}^{\infty} \mu_{n} \exp (i n \phi) \int_{0}^{\tau} d s g(s) \exp (\text { in } \omega s)
\end{aligned}
$$

where in writing Eq.(3.39) and (3.40) we have used Eq.(3.17). For the second iteration we put Eq.(3.39) and (3.40) back into Eq.(3.36) and (3.37) and thus obtain $\Delta J_{t}^{(2)}(\tau)$ and $\Delta \phi_{t}^{(2)}(\tau)$. Putting them back into Eq.(3.36) and (3.37) again we obtain $\Delta J_{t}^{(3)}(\tau)$ and $\Delta \phi_{t}^{(3)}(\tau)$. These are presented in some details in the Appendix.

In calculating the moments as demanded by Eq.(3.25), we have neglected all higher powers $(n \geq 2)$ of $\tau$ and $1 / \omega$. The reason for doing this in case of $\tau$ is clear from the limit imposed on $\tau$ in Eq.(3.25). For $1 / \omega$ also, this approximation is legitimate since $1 / \omega$ is the shortest time scale of the problem (see inequality (3.7)). The final results for the moments are:

$$
\begin{aligned}
\left\langle\left[\Delta J_{t}(\tau)\right]^{2}\right\rangle_{S} & =4 \tau \sum_{n=1}^{\infty} n^{2}\left|x_{n}\right|^{2} \tilde{C}_{n}^{c}(\omega) \\
\left\langle\left[\Delta \phi_{t}(\tau)\right]^{2}\right\rangle_{S} & =4 \tau \sum_{n=1}^{\infty}\left|\frac{d x_{n}}{d J}\right|^{2} \tilde{C}_{n}^{c}(\omega) \\
\left\langle\left[\Delta J_{t}(\tau)\right]\left[\Delta \phi_{t}(\tau)\right]\right\rangle_{S} & =0 \\
\left\langle\Delta J_{t}(\tau)\right\rangle_{S} & =-2 \tau \sum_{n=1}^{\infty} n^{2}\left[\omega\left|x_{n}\right|^{2} \tilde{\beta}_{n}^{c}(\omega)-\frac{d}{d J}\left\{\left|x_{n}\right|^{2} \tilde{C}_{n}^{c}(\omega)\right\}\right] \\
\left\langle\Delta \phi_{t}(\tau)\right\rangle_{S} & =\omega \tau+\tau \sum_{n=1}^{\infty} n\left[\omega \tilde{\beta}_{n}^{s} \frac{d\left|x_{n}\right|^{2}}{d J}-\frac{d}{d J}\left(\tilde{C}_{n}^{s} \frac{d\left|x_{n}\right|^{2}}{d J}\right)\right]-\tau f_{0}^{\prime} \mu_{0} t_{c}
\end{aligned}
$$

where

$$
\begin{aligned}
\tilde{\beta}_{n}^{c} & =\int_{0}^{\infty} d t \beta(t) \cos (n \omega t) \\
\tilde{\beta}_{n}^{s} & =\int_{0}^{\infty} d t \beta(t) \sin (n \omega t) \\
\tilde{C}_{n}^{c} & =\int_{0}^{\infty} d t C(t) \cos (n \omega t) \\
\tilde{C}_{n}^{s} & =\int_{0}^{\infty} d t C(t) \sin (n \omega t)
\end{aligned}
$$

and

$$
f_{0}^{\prime}=-\sum_{n=3}^{\infty} \frac{1}{(n-1) !} \frac{\partial}{\partial t}\left[V^{n}[x(t)]\left\{\left\langle\delta \hat{X}^{n-1}(t)\right\rangle-\left\langle\delta \hat{X}^{n-1}(0)\right\rangle\right\}\right]
$$

Also

$$
\begin{aligned}
\tilde{\beta}_{n}(\omega) & =\tilde{\beta}_{n}^{c}(\omega)-i \tilde{\beta}_{n}^{s}(\omega) \\
\tilde{C}_{n}(\omega) & =\tilde{C}_{n}^{c}(\omega)-i \tilde{C}_{n}^{s}(\omega)
\end{aligned}
$$

Some remarks are needed in connection with Eq.(3.41) to Eq.(3.45). Let us now examine how the quantum notion is implied in Eqs.(3.41 3.45). First, all the moments are the functions of the Fourier components $x_{n}(J)$ where $J$ is a 
quantum $c$-number. Second, the moments are crucially dependent on the Fourier components of quantum correlation function $C(t)$ of the heat bath. In the classical limit $x_{n}(J)$ becomes the functions of the classical action variable $J$ and also $\tilde{C}_{n}(\omega)$ reduces to $\tilde{C}_{n}(\omega)=k_{B} T \tilde{\beta}_{n}(\omega)$. We thus obtain,

$$
\begin{aligned}
& \left\langle\left[\Delta J_{t}(\tau)\right]^{2}\right\rangle_{S}=4 \tau k_{B} T \sum_{n=1}^{\infty} n^{2}\left|x_{n}\right|^{2} \tilde{\beta}_{n}^{c}(\omega) \\
& \left\langle\left[\Delta \phi_{t}(\tau)\right]^{2}\right\rangle_{S}=4 \tau k_{B} T \sum_{n=1}^{\infty}\left|\frac{d x_{n}}{d J}\right|^{2} \tilde{\beta}_{n}^{c}(\omega)
\end{aligned}
$$

in the high-temperature limit $\hbar \omega \ll k_{B} T$. The last term in Eq.(3.45) is due to a correction to frequency $\omega$ and is of pure quantum origin (3.1f). $f_{0}^{\prime}$ is precisely the co-efficient of $t$ in the Taylor expansion of Eq.(3.1f). $t_{c}$ is the 'cut-off' time upto which the quantum fluctuation remains linear in time and is approximated as $\sim 1 / \omega$, as allowed by the time scale of the problem. Thus the quantum character of the nonlinear system enters into the description in two different ways. First, classical Hamiltonian gets modified by quantum corrections at $t=0$ (see Eq.(3.3)). This makes action angle variables bear quantum signature. Second, the quantum correction for $t>0$ as contained in $g(t)$ makes its presence in phase drift term in (3.45).

Inserting Eq.(3.41) to (3.45) in Eq.(3.25) and thereby disregarding terms with $n>2$ with the following definitions

$$
\begin{aligned}
\epsilon(J) & =2 \sum_{n=1}^{\infty} n^{2}\left|x_{n}\right|^{2} \tilde{\beta}_{n}^{c}(\omega) \\
\Gamma(J) & =2 \sum_{n=1}^{\infty}\left|\frac{d x_{n}}{d J}\right|^{2} \tilde{C}_{n}^{c}(\omega) \\
\Omega(J) & =\omega+\sum_{n=1}^{\infty} n\left[\omega \tilde{\beta}_{n}^{s} \frac{d\left|x_{n}\right|^{2}}{d J}-\frac{d}{d J}\left(\tilde{C}_{n}^{s} \frac{d\left|x_{n}\right|^{2}}{d J}\right)\right]-f_{0}^{\prime} \mu_{0} t_{c}
\end{aligned}
$$

we obtain the Fokker-Planck equation for $P(J, \phi, t)$ as,

$$
\begin{aligned}
\frac{\partial P(J, \phi, t)}{\partial t} & =\frac{\partial}{\partial J}\left[2 \sum_{n=1}^{\infty} n^{2}\left|x_{n}\right|^{2} \tilde{\beta}_{n}^{c}(\omega)\left\{\frac{\tilde{C}_{n}^{c}(\omega)}{\tilde{\beta}_{n}^{c}(\omega)} \frac{\partial}{\partial J}+\omega(J)\right\} P\right] \\
& +\Gamma(J) \frac{\partial^{2} P}{\partial \phi^{2}}-\Omega(J) \frac{\partial P}{\partial \phi} .
\end{aligned}
$$

If the distribution function is initially independent of $\phi$ it satisfies the quantum diffusion equation in action space

$$
\frac{\partial P(J, t)}{\partial t}=\frac{\partial}{\partial J}\left[\epsilon(J)\left\{\Lambda \frac{\partial}{\partial J}+\omega(J)\right\} P\right]
$$

where by virtue of $(3.5 d)$ we write

$$
\begin{aligned}
\Lambda & =\Lambda(\bar{\omega}) \simeq \frac{\tilde{C}_{n}^{c}(\bar{\omega})}{\tilde{\beta}_{n}^{c}(\bar{\omega})} \\
\text { or } \quad \Lambda & =\hbar \bar{\omega}[\bar{n}(\bar{\omega})+1 / 2]
\end{aligned}
$$

Here $\bar{\omega}$ is the linearized frequency and $\Lambda$ plays the typical role of $k_{B} T$. We have

$$
\omega(J)=\frac{\partial H}{\partial J}=\frac{d E}{d J} .
$$

Expressing

$$
\omega(J)=\nu(E)
$$

we have

$$
\frac{\partial}{\partial J}=\nu(E) \frac{\partial}{\partial E}
$$


With this transformation the quantum Kramers' equation for energy diffusion [Eq.(3.52)] looks like,

$$
\frac{\partial P(E, t)}{\partial t}=\frac{\partial}{\partial E}\left[D(E)\left(\frac{\partial}{\partial E}+\frac{1}{\Lambda}\right) \nu(E) P(E, t)\right]
$$

where the diffusion coefficient is given by

$$
D(E)=\nu(E) 2 \hbar \bar{\omega}\left[\bar{n}(\bar{\omega})+\frac{1}{2}\right] \sum_{n=1}^{\infty} n^{2}\left|x_{n}\right|^{2} \int_{0}^{\infty} d t \beta(t) \cos [n \nu(E) t] .
$$

$E q .(3.50)$ is the first key result of the present paper. The equation is valid for arbitrary temperature and noise correlation. The prime quantities that determine the equation for energy diffusion (3.56) are the diffusion coefficients $D$, the quantum analogue of $k T, \Lambda$ and the frequency of the dynamical system, $\nu(E)$. It is important to note that all the quantities as defined by (3.57), (3.53) and (3.54), respectively contain quantum contributions. In the classical limit Eq. (3.53) reduces to $k T$ when $\bar{n}(\bar{\omega}) \gg 1 / 2$ and $\bar{n}(\bar{\omega})\left(=[\exp (\hbar \bar{\omega} / k T)-1]^{-1}\right) \approx k T / \hbar \bar{\omega}$. Since by virtue (3.54) $\nu(E)=\omega(J)=\partial H / \partial J$ with $H$ defined as $H=(1 / 2) v^{2}+V_{q}(x)$ where $V_{q}(x)$ includes quantum corrections over the classical potential $V(x)$ according to Eq.(3.3), $\nu(E)$ reduces to classical frequency in the classical limit as $\hbar \rightarrow 0$. Although the expression for the diffusion coefficient (3.57) looks a bit complicated and formal due to the appearence of the Fourier coefficients $x_{n}$ in the summation, it is possible to read the various terms in $D(E)$ in the following way. $D(E)$ is essentially an approximate product of three terms, $\hbar \bar{\omega}[\bar{n}(\bar{\omega})+1 / 2], \int_{0}^{\infty} d t \beta(t) \cos [n \nu(E) t]$ and $\nu(E) \sum_{n=1}^{\infty} n^{2}\left|x_{n}\right|^{2}$, where the $n$ dependence of the latter two contributions have been separated out for interpretation. The integral is the Fourier transform of the memory kernel, while the sum can be shown to be equal to $J$ ( Appendix D of Ref. [10]), which is the quantum action variable. In the classical limit the quantum diffusion coefficient $D(E)$ therefore clearly reduces to the classical diffusion coefficient of Carmeli and Nitzan [10]. A few further remarks on the related issues may be made at this point. To address the problem of nonequilibrium quantum tunneling above cross-over temperature (nonequilibrium situation arises due to the significant growth of population above zero levels at temperature above cross-over since the dissipation is very weak), several authors [38 43] have advocated the use of a probability function per unit time (of finding the system in the barrier region near a classical turning point with energy $E$ ) which obeys an integral equation 43 whose the differential approximation leads to an equation similar (not the same) to Eq.(3.56). The notable difference is in the fact that the former equation is applicable above cross-over temperature while Eq.(3.56) works at all temperature down to vacuum limit. The energy loss coefficient in (3.56), i.e., $D(E) / \Lambda$ when put into the form [ using (3.53) ],

$$
\frac{D(E)}{\Lambda}=\int_{0}^{\infty} d t \beta(t) \nu(E) \sum_{n=1}^{\infty} 2 n^{2}\left|x_{n}\right|^{2} \cos [n \nu(E) t]
$$

is comparable to that of Griff et al [43], the integrand without $\beta(t)$ being a function of action (or equivalently energy). These results can be utilized as a consistency check of the present scheme.

\section{QUANTUM ENERGY DIFFUSION CONTROLLED RATE OF ESCAPE}

The classical treatment of memory effects in the energy diffusion controlled escape is now well-documented in the literature [10, 35] [36]. To address the corresponding problem in the quantum domain we start by recasting the Kramers' equation in the energy diffusion regime [Eq.(3.52)] in the form of a continuity equation to obtain,

$$
\frac{\partial P(E, t)}{\partial t}+\frac{\partial j_{E}}{\partial E}=0
$$

where $j_{E}$ is the flux along the energy co-ordinate at thermal equilibrium and is given by,

$$
j_{E}=-D(E)\left[\frac{\partial}{\partial E}+\frac{1}{\Lambda}\right] \nu(E) P_{s t}(E)
$$

where $P_{s t}$ is the stationary probability distribution. For zero current condition, we have the equilibrium distribution, $P_{e q}$ at the source well as, 


$$
\begin{aligned}
P_{e q}(E) & =\frac{N^{-1}}{\nu(E)} \exp (-E / \Lambda) \\
& =\frac{N^{-1}}{\nu(E)} \exp \left[-\left(E^{c}+\Delta Q\right) / \Lambda\right]
\end{aligned}
$$

where we have split the energy into classical $\left(E^{c}\right)$ and quantum $(\Delta Q)$ parts, the contribution arising from the latter being very small. We now define the rate of escape $k$ as flux over population

$$
k=\frac{j_{E}}{n_{a}}
$$

where

$$
\begin{aligned}
n_{a} & =\text { total population at the source well } \\
& =\int_{0}^{E_{b}^{c}} P(E) d E
\end{aligned}
$$

Here $E_{b}^{c}$ is the classical value of the activation barrier. Following BHL 448] we use a Kramers' like ansatz

$$
P(E)=\eta(E) P_{e q}(E)
$$

to arrive at

$$
j_{E}=-D(E) \nu(E) P_{e q}(E) \frac{\partial \eta(E)}{\partial E}
$$

Integrating the above expression from $E=E_{1} \simeq \Lambda$ (see Eq.(3.53) ) to $E=E_{b}^{c}$, one derives an expression for energy independent current $j_{E}$ (with $E \leq E_{b}^{c}$ ) as,

$$
\begin{aligned}
j_{E} & =\frac{\left[\eta(\Lambda)-\eta\left(E_{b}^{c}\right)\right]}{\int_{\Lambda}^{E_{b}^{c}} \frac{d E}{D(E) \nu(E) P_{e q}(E)}} \\
& =\left[1-\eta\left(E_{b}^{c}\right)\right] D\left(E_{b}^{c}\right) \frac{N^{-1}}{\Lambda} e^{-E_{b}^{c} / \Lambda}
\end{aligned}
$$

where we have used the boundary condition $\eta(\Lambda) \simeq 1$.

Following the original reasoning by BHL we now allow an outflow $j_{\text {out }}$ from each energy range $E$ to $E+d E$, with each $E$ satisfying the condition $E \geq E_{b}^{c}$. Then we can write,

$$
d j_{\text {out }}=\alpha \nu(E) \eta(E) P_{e q}(E) d E
$$

which is compensated by a divergence in the vertical flow,

$$
\frac{d j_{E}}{d E}=-\alpha \nu(E) \eta(E) P_{e q}(E)
$$

Here $\alpha$ is a parameter which has been set approximately equal to one by BHL. Inserting the expression for nonequilibrium current Eq.(4.7), we obtain the ordinary differential equation for $\eta(E)$ as,

$$
D(E) \frac{d^{2} \eta}{d E^{2}}+\left[\frac{d D(E)}{d E}-D(E) \frac{1}{\Lambda}\right] \frac{d \eta}{d E}-\alpha \eta(E)=0 .
$$

Within small energy range above $E_{b}^{c}$ one can assume essentially a constant diffusion co-efficient i.e.,

$$
\left[\frac{d D(E)}{d E}\right]_{E \simeq E_{b}^{c}}=0 \text { for } E \geq E_{b}^{c}
$$

Substituting a trial solution of the form $\eta(E)=C \exp (s E / \Lambda)$ for $s<0$, in Eq.(4.11) we have,

$$
s_{-}=-\frac{1}{2}\left[\left(1+\frac{4 \alpha \Lambda^{2}}{D\left(E_{b}^{c}\right)}\right)^{1 / 2}-1\right] .
$$


Setting $\eta(E)=\eta\left(E_{b}^{c}\right) \exp \left[s\left(E-E_{b}^{c}\right) / \Lambda\right]$ and putting this into Eq.4.7) and comparing this with the right hand side of Eq.(4.8) we have,

$$
\eta\left(E_{b}^{c}\right)=1 /(1-s) \text { for } s<0
$$

Thus, escape rate $k$ can be obtained as,

$$
k=j_{E}\left[\int_{0}^{E_{b}^{c}} \eta(E) P_{e q}(E) d E\right]^{-1} .
$$

Making use of (4.14) in (4.8) and the resulting expression for $j$ in (4.15) we obtain

$$
k=\frac{-s}{1-s}\left[\frac{\int_{0}^{E_{b}^{c}} \eta(E) P_{e q}(E) d E}{\left(N^{-1} / \Lambda\right) D\left(E_{b}^{c}\right) \exp \left(-E_{b}^{c} / \Lambda\right)}\right]^{-1} .
$$

For the dynamics at the bottom we have $\eta \rightarrow 1$. Recalling that $E=E^{c}+\Delta Q$, where $\Delta Q$ is the quantum contribution to classical energy, we expand $E$ in a Taylor series. Here $E^{c}=\left(v^{2} / 2\right)+V(x)$ and $\Delta Q(x)$ is the quantum correction terms in (3.3). Retaining terms upto the second order in $x$, and making harmonic approximation around the bottom of the well at $x=0$, we get

$$
E=\frac{p^{2}}{2}+\frac{1}{2} \omega_{0}^{2} x^{2}+\Delta Q_{0}+\Delta Q_{0}^{\prime} x+\frac{1}{2} \Delta Q_{0}^{\prime \prime} x^{2}
$$

where $\omega_{0}$ corresponds to the frequency at the bottom of the classical potential $V(x)$ at $x=0$ so that $\omega_{0}^{2}=$ $\partial^{2} V(x) /\left.\partial x^{2}\right|_{x=0}$. The subscript zero in $\Delta Q_{0}, \Delta Q_{0}^{\prime}$ and $\Delta Q_{0}^{\prime \prime}$ are the quantities evaluated at this point.

Now $n_{a}$, the total population at the source well, can be evaluated as,

$$
\begin{aligned}
n_{a} & =\int_{-\infty}^{\infty} \int_{-\infty}^{\infty} P_{e q}(E) d x d p \\
& =\exp \left[-\Delta Q_{0} / \Lambda\right] \int_{-\infty}^{\infty} \exp \left(-v^{2} / 2 \Lambda\right) d v \int_{-\infty}^{\infty} \exp \left[-\frac{1}{\Lambda}\left(\frac{1}{2} \omega_{0}^{2} x^{2}+\Delta Q_{0}^{\prime} x+\frac{1}{2} \Delta Q_{0}^{\prime \prime} x^{2}\right)\right] d x .
\end{aligned}
$$

Thus

$$
n_{a}=\frac{1}{N} \frac{2 \pi \Lambda}{\sqrt{\omega_{0}^{2}+\Delta Q_{0}^{\prime \prime}}} \exp \left[-\frac{\Delta Q_{0}}{\Lambda}+\frac{\left(\Delta Q_{0}^{\prime}\right)^{2}}{2 \Lambda\left(\omega_{0}^{2}+\Delta Q_{0}^{\prime \prime}\right)}\right] .
$$

So, the quantum non-Markovian rate of escape from a metastable well in the low-friction regime is given by,

$$
\begin{aligned}
k= & {\left[\frac{\left\{1+\left(4 \alpha \Lambda^{2}\right) / D\left(E_{b}^{c}\right)\right\}^{1 / 2}-1}{\left\{1+\left(4 \alpha \Lambda^{2}\right) / D\left(E_{b}^{c}\right)\right\}^{1 / 2}+1}\right] \frac{D\left(E_{b}^{c}\right)}{\Lambda^{2}} \frac{\sqrt{\omega_{0}^{2}+\Delta Q_{0}^{\prime \prime}}}{2 \pi} } \\
& \times \exp \left[-\frac{1}{\Lambda}\left\{E_{b}^{c}-\Delta Q_{0}+\frac{\left(\Delta Q_{0}^{\prime}\right)^{2}}{2\left(\omega_{0}^{2}+\Delta Q_{0}^{\prime \prime}\right)}\right\}\right] .
\end{aligned}
$$

The above expression is the second key result of the paper. It has the form of the celebrated Arrhenius expression for rate coefficient with the classical activation energy $E_{b}^{c}$ in the exponential factor and a complicated $\Lambda$ and $D$ dependent quantity in the pre-exponential factor. As noted earlier in the detailed discussion of quantum diffusion coefficient in the context of Eq.(3.56), the diffusion coefficient $D(E)$ is contributed by the three factors and has to be evaluated at the barrier top. The main effect of the pre-exponential factor is that the rate becomes proportional to the damping coefficient and the memory kernel results in the decrease of pre-factor for increasing correlation time. The structure of the rate expression (4.19) suggests that it has the same form of the pre-exponential factor as that of Hänggi and Weiss [35] although its content is quantum mechanical in character. The quantum mechanical content of the rate expression lies in several quantities, e.g., quantum diffusion coefficient $D\left(E_{b}^{c}\right)$, quantum analogue of $k_{B} T, \Lambda$ as given by (3.57) and (3.53), respectively. The frequency at the bottom of the well, $\omega_{0}$ as well as the classical activation energy $E_{b}^{c}$ get modified by quantum correction $\Delta Q_{0}^{\prime \prime}$ and $\Delta Q_{0}$ terms. The result of Hänggi and Weiss [35] for the classical non-Markovian case can then be appropriately recovered. It is thus apparent that quantum correction terms in the exponential factor is (4.19) depends on the nature of the potential which in turn determines the rate. In what follows in the next section we illustrate the results with a specific cubic potential of Kramers' form. We mention in passing that throughout the treatment the noise intensity needs to be small for the result (4.19) to be a good description of the activated process controlled by energy diffusion. 


\section{AN EXAMPLE WITH CUBIC POTENTIAL}

We consider a model cubic potential of the form $V(\hat{X})=-(1 / 3) A \hat{X}^{3}+B \hat{X}^{2} . A$ and $B$ are two constant parameters of the problem with $A>0$ and $B>0$. Then by virtue of Eq.(3.3) we have the $c$-number form of quantum potential

$$
V_{q}(x)=-\frac{A}{3} x^{3}+B x^{2}-A\left\langle\delta \hat{X}^{2}(0)\right\rangle x+\text { constant }
$$

so that the time independent Hamiltonian is given by,

$$
H(x, v)=\frac{v^{2}}{2}-\frac{A}{3} x^{3}+B x^{2}-C x=E
$$

where $C=A \hbar /(2 \sqrt{2 B})$. We have used $\left\langle\delta \hat{X}^{2}(0)\right\rangle=\hbar /(2 \sqrt{2 B})$, the minimum uncertainty, and ignored the constant part in $V_{q}(x)$. Here $C$ refers to quantum contribution to classical potential due to which the minimum, the metastable point corresponding to $V_{q}(x)$ shifts to $x_{0}=C /(2 B)$ (with respect to the corresponding classical metastable minimum at $x=0)$.

Linearizing the potential $V_{q}(x)$ around $x_{0}$ we obtain

$$
V_{q}(x)=V_{q}\left(x_{0}\right)+\left(B-\frac{A C}{2 B}\right)\left(x-x_{0}\right)^{2} .
$$

We then calculate the action $J$, the usual form of which is given by,

$$
J=2 \int_{x_{1}}^{x_{2}} v d x
$$

$x_{1}$ and $x_{2}$ are the two turning points of oscillation for which $v$ is equal to zero and they jointly correspond to a particular value of the system energy $E$. In principle, they are the first two roots (in ascending order of magnitude) of the cubic equation

$$
\frac{A}{3} x^{3}-B x^{2}+C x+E=0
$$

the third root being irrelevant for the present purpose. $x_{1}$ and $x_{2}$ however can be approximately calculated by simply putting $V_{q}(x)=E$ (since $v=0$ is the turning point) in (5.3) and solving the resulting equation for $x$.

$$
x_{1,2} \simeq x_{0} \mp\left(\frac{E-V_{q}\left(x_{0}\right)}{B}\right)^{1 / 2} .
$$

Putting the value of $v$ from Eq.(5.2) in Eq.(5.4) we get the action integral in the form,

$$
J=2 \sqrt{2} \int_{x_{1}}^{x_{2}}\left[\left(E-B x^{2}\right)+\left(\frac{A}{3} x^{3}+C x\right)\right]^{1 / 2} d x .
$$

Putting Eq.(3.54) in Eq.(3.57) we can express the quantum diffusion co-efficient in terms of the action as,

$$
\tilde{D}(J)=\omega(J) 2 \hbar \bar{\omega}_{0}\left[\bar{n}\left(\bar{\omega}_{0}\right)+\frac{1}{2}\right] \sum_{n=1}^{\infty} n^{2}\left|x_{n}\right|^{2} \int_{0}^{\infty} d t \beta(t) \cos [n \omega(J) t]
$$

where we have replaced $D(E)$ by $\tilde{D}(J)$ to emphasize the change made in the argument. Furthermore for unit mass of the Brownian particle we may write [10]

$$
\omega(J) \sum_{n=-\infty}^{\infty} n^{2}\left|x_{n}(J)\right|^{2}=J
$$

Putting Eq. (5.9) in Eq.(5.8) the diffusion coefficient can be approximately expressed as

$$
\tilde{D}(J) \simeq 2 J \hbar \bar{\omega}_{0}\left[\bar{n}\left(\bar{\omega}_{0}\right)+\frac{1}{2}\right] \int_{0}^{\infty} d t \beta(t) \cos [n \omega(J) t] .
$$


For the present form of model potential we also have $\Delta Q(x)=-C x$ for which $\Delta Q_{0}=0, \Delta Q_{0}^{\prime \prime}=0$ and $\Delta Q_{0}^{\prime}=-C$. With these expressions for quantum contributions and making use of Eq.(5.10) in Eq.(4.19) we have the final expression for the escape rate as,

$$
k=\frac{\omega_{0}}{2 \pi}\left[\frac{\left\{1+\left(4 \alpha \Lambda^{2}\right) / \tilde{D}\left(J_{b}\right)\right\}^{1 / 2}-1}{\left\{1+\left(4 \alpha \Lambda^{2}\right) / \tilde{D}\left(J_{b}\right)\right\}^{1 / 2}+1}\right] \frac{\tilde{D}\left(J_{b}\right)}{\Lambda^{2}} \exp \left[-\frac{1}{\Lambda}\left\{E_{b}^{c}+\frac{A^{2} \hbar^{2}}{4 \omega_{0}^{3}}\right\}\right]
$$

Here $J_{b}$ denotes the value of the action of the system at the barrier top. It should be noted that it includes both the classical and quantum contributions. $E_{b}^{c}$ corresponds to classical activation energy which gets modified by a contribution due to quantum correction entangled with the nonlinearity of the potential. It is important to note that the positivity of the factor $\left(A^{2} \hbar^{2}\right) /\left(4 \omega_{0}^{3} \Lambda\right)$ in the exponential in (5.11) results in a larger effective activation barrier which causes a net reduction of the full rate below its corresponding classical value. This is in good agreement with the earlier observation by Griff et al [43] and is somewhat counterintuitive - as emphasized by Hänggi et al [4] to the fact that full rate comprises classical rate plus zero temperature tunneling. The quantum reduction of total rate in the weak friction regime is a manifestation of interplay of thermal noise and quantum fluctuation and is expected to be pronounced for systems with flat barriers, commonly encountered in absorption-desorption processes in surface

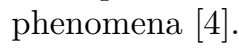

\section{CONCLUSIONS}

Based on a true quantum phase space distribution function and an ensemble average procedure we have derived a generalized Kramers' equation for energy diffusion and analyzed the quantum transmission coefficient associated with the rate coefficient within a full quantum mechanical framework in the low friction regime. The present formulation is a complementary follow-up to our recent work [32 on quantum Kramers' theory in the spatial diffusion limited regime. The main conclusions of this study are the following:

(i) The proposed Kramers' equation in the energy diffusion regime is an exact quantum analogue of non-Markovian classical Kramers' equation derived by $\mathrm{CN}$ [10] in eighties. The equation retains its full validity both in the classical and vacuum limits at arbitrary temperature and noise correlation of the heat bath.

(ii) The generalized quantum rate coefficient for the decay from a metastable well reduces to Kramers'-Hänggi-Weiss rate [35] in the classical limit and to pure weak dissipative tunneling rate in the quantum limit at zero temperature.

(iii) While in the intermediate to strong damping regime the total Kramers' rate comprising classical as well as quantum rate is always higher than the corresponding classical rate, the notable feature in the weak friction regime ( for a metastable potential ) is a net quantum reduction of the total rate below its corresponding classical value. This is in conformity with the earlier observation in this context 433.

(iv) While the existing methods of calculation of quantum Kramers' rate is based on path integral techniques [4, 21 23, 60, 61, we rely on a canonical quantization procedure and true probability distribution function of $c$-number variables. To the best of our knowledge the implementation of a differential equation and its solution as a boundary value problem have not been tried up to date for quantum Kramers' problem. The methodology as pursued here allows us to apply classical techniques for the quantum problem of barrier crossing dynamics.

(v) The quantum effects appear in the present formulation in two different ways. The nonlinear part of the potential of the system gives rise to quantum dispersion, while the heat bath imparts quantum noise. An important advantage of the present method is that it is possible to incorporate quantum corrections to all orders and one need not invoke any semiclassical technique which is almost always used in the practical evaluation of the formal functional integrals.

The present scheme of mapping of the quantum theory of Brownian motion in energy space into a classical form offers an opportunity to generate quantum noise as classical $c$-numbers and study numerically the quantum stochastic dynamics independent of path integral Monte Carlo techniques [62 64. We hope to address this issue in a future communication.

\section{ACKNOWLEDGMENTS}

The authors are indebted to the Council of Scientific and Industrial Research (CSIR), Government of India, for financial support under grant No. 01/(1740)/02/EMR-II. 


\section{APPENDIX A: CALCULATION OF MOMENTS}

Some details of the calculations involving the iterations (upto the third order) for determination of the moments have been shown here. The procedure followed here is that of classical theories of Carmeli and Nitzan [10]. We have stressed the steps for which the quantum contributions form essentially new content.

\section{First Iteration}

Inserting Eq.(3.38a) and Eq. 3.38b) in Eq.3.36) we have,

$$
\begin{aligned}
& \Delta J_{t}^{(1)}(\tau)=-\sum_{n=-\infty}^{\infty} \sum_{m=-\infty}^{\infty} B_{n m} \exp [i(n+m) \phi] \int_{0}^{\tau} d s \exp [i(n+m) \omega s] \\
& \quad+\sum_{n=-\infty}^{\infty} \sigma_{n} \exp (i n \phi) \int_{0}^{\tau} d s F(s) \exp (i n \omega s)+\sum_{n=-\infty}^{\infty} \sigma_{n} \exp (i n \phi) \int_{0}^{\tau} d s g(s) \exp (\text { in } \omega s)
\end{aligned}
$$

where we have suppressed the arguments of quantities $B_{n m}, \sigma_{n}$ and $\phi$ for the sake of brevity. Now, from Eq.(3.7) and the argument following Eq.(3.25) we can infer that $\omega \tau \gg 1$. So we can write

$$
\begin{aligned}
\int_{0}^{\tau} d s \exp [i(n+m) \omega s] & \simeq \tau \delta_{n,-m} \\
\text { for which } \quad \sum_{n, m=-\infty}^{\infty} B_{n m} \exp [i(n+m)] \tau \delta_{n,-m} & =\tau \sum_{n=-\infty}^{\infty} B_{n,-n}
\end{aligned}
$$

Thus,

$$
\begin{aligned}
\Delta J_{t}^{(1)}(\tau)= & -\tau \sum_{n=-\infty}^{\infty} B_{n,-n}+\sum_{n=-\infty}^{\infty} \sigma_{n} \exp (i n \phi) \int_{0}^{\tau} d s F(s) \exp (i n \omega s) \\
& +\sum_{n=-\infty}^{\infty} \sigma_{n} \exp (i n \phi) \int_{0}^{\tau} d s g(s) \exp (i n \omega s)
\end{aligned}
$$

Similarly from Eq.(3.37) we have

$$
\begin{aligned}
\Delta \phi_{t}^{(1)}(\tau)= & \omega \tau+\tau \sum_{n=-\infty}^{\infty} C_{n,-n}-\sum_{n=-\infty}^{\infty} \mu_{n} \exp (i n \phi) \int_{0}^{\tau} d s F(s) \exp (i n \omega s) \\
& -\sum_{n=-\infty}^{\infty} \mu_{n} \exp (i n \phi) \int_{0}^{\tau} d s g(s) \exp (i n \omega s)
\end{aligned}
$$

Along with these we will also require the statistical averages of the above expressions for calculation of the moments. Thus,

$$
\left\langle\Delta J_{t}^{(1)}(\tau)\right\rangle_{S}=-\tau \sum_{n=-\infty}^{\infty} B_{n,-n}+\sum_{n=-\infty}^{\infty} \sigma_{n} \exp (i n \phi) \int_{0}^{\tau} d s g(s) \exp (\text { in } \omega s)
$$

where we have used (2.10). We can proceed further with Eq.(A6) and cast it in a more transparent form. In this context it is worth mentioning that so far as the quantum noise $g(t)$ is concerned, we take only the significant terms from Eq.(3.1f) which result in terms linear in $\tau$. Referring back to Eq.(3.32) we then have,

$$
\begin{aligned}
-\tau \sum_{n=-\infty}^{\infty} B_{n,-n} & =-\tau \sum_{n=-\infty}^{\infty} n^{2} \omega\left|x_{n}\right|^{2} \tilde{\beta}_{n}(\omega) \\
& =-2 \tau \sum_{n=1}^{\infty} n^{2} \omega\left|x_{n}\right|^{2} \tilde{\beta}_{n}^{c}(\omega)
\end{aligned}
$$


using Eq.(3.46a). The second expression of Eq. A6 has been shown to be negligible in Appendix-B. Thus

$$
\left\langle\Delta J_{t}^{(1)}(\tau)\right\rangle_{S}=-2 \tau \sum_{n=1}^{\infty} n^{2} \omega\left|x_{n}\right|^{2} \tilde{\beta}_{n}^{c}(\omega)
$$

Similarly from Eq.(A5) we have,

$$
\left\langle\Delta \phi_{t}^{(1)}(\tau)\right\rangle_{S}=\omega \tau+\tau \sum_{n=1}^{\infty} n \omega \frac{d\left|x_{n}\right|^{2}}{d J} \tilde{\beta}_{n}^{s}-\tau f_{0}^{\prime} \mu_{0} t_{c}
$$

In deriving Eq. (A9) we have used Eq. (3.31) and (3.33). Otherwise, the way leading to Eq. (A9) is similar to that of Eq.(A8). The origin of the last term is the same integral that appears in Eq.(A6) and is shown in Appendix-B.

\section{Second Iteration}

Here we insert Eq. A44) and (A5) into the right hand side's of Eq.(3.36) and Eq.(3.37). Keeping in mind Eq.(3.38a $)$ and $(3.38 \mathrm{~b})$ we expand the functions of $\Delta J_{t}(s)$ and $\Delta \phi_{t}(s)$, viz. $B_{n m}, C_{n m}, \sigma_{n}$ and $\mu_{n}$, keeping terms upto the first order only. Thus,

$$
\begin{aligned}
B_{n m}\left[J(t)+\Delta J_{t}(s)\right] & =B_{n m}+B_{n m}^{\prime} \Delta J_{t}(s) \\
\text { with } \quad B_{n m} & =B_{n m}[J(t)] \\
\text { and } \quad B_{n m}^{\prime} & =\left[\frac{d B_{n m}}{d J}\right]_{J=J(t)}
\end{aligned}
$$

The expansion has been done about $\Delta J_{t}^{(0)}(s)=0$ and $\Delta \phi_{t}^{(0)}(s)=\omega s$. The same expansion procedure is followed for $C_{n m}, \sigma_{n}, \mu_{n}$ and the exponentials as well, occurring in Eq.(3.36) and (3.37). We therefore obtain,

$$
\begin{aligned}
& \Delta J_{t}^{(2)}(\tau)=\Delta J_{t}^{(1)}(\tau)-\sum_{n=-\infty}^{\infty} \sum_{m=-\infty}^{\infty} \sum_{l=-\infty}^{\infty}\left[B_{n m}^{\prime} \sigma_{l}-i(n+m) B_{n m} \mu_{l}\right] \exp [i(n+m+l) \phi] \\
& \quad \times \int_{0}^{\tau} d s \int_{0}^{s} d s_{1} F\left(s_{1}\right) \exp \left[i(n+m) \omega s+i l \omega s_{1}\right]-\sum_{n=-\infty}^{\infty} \sum_{m=-\infty}^{\infty} \sum_{l=-\infty}^{\infty}\left[B_{n m}^{\prime} \sigma_{l}-\right. \\
& \left.\quad i \quad(n+m) B_{n m} \mu_{l}\right] \exp [i(n+m+l) \phi] \int_{0}^{\tau} d s \int_{0}^{s} d s_{1} g\left(s_{1}\right) \exp \left[i(n+m) \omega s+i l \omega s_{1}\right] \\
& \quad+\sum_{n=-\infty}^{\infty} \sum_{l=-\infty}^{\infty}\left(\sigma_{n}^{\prime} \sigma_{l}-i n \sigma_{n} \mu_{l}\right) \exp [i(n+l) \phi] \int_{0}^{\tau} d s \int_{0}^{s} d s_{1} F(s) F\left(s_{1}\right) \exp \left(i n \omega s+i l \omega s_{1}\right) \\
& \quad+\sum_{n=-\infty}^{\infty} \sum_{l=-\infty}^{\infty}\left(\sigma_{n}^{\prime} \sigma_{l}-i n \sigma_{n} \mu_{l}\right) \exp [i(n+l) \phi] \int_{0}^{\tau} d s \int_{0}^{s} d s_{1} F(s) g\left(s_{1}\right) \exp \left(i n \omega s+i l \omega s_{1}\right) \\
& \quad+\sum_{n=-\infty}^{\infty} \sum_{l=-\infty}^{\infty}\left(\sigma_{n}^{\prime} \sigma_{l}-i n \sigma_{n} \mu_{l}\right) \exp [i(n+l) \phi] \int_{0}^{\tau} d s \int_{0}^{s} d s_{1} g(s) F\left(s_{1}\right) \exp \left(i n \omega s+i l \omega s_{1}\right) \\
& \quad+\sum_{n=-\infty}^{\infty} \sum_{l=-\infty}^{\infty}\left(\sigma_{n}^{\prime} \sigma_{l}-i n \sigma_{n} \mu_{l}\right) \exp [i(n+l) \phi] \int_{0}^{\tau} d s \int_{0}^{s} d s_{1} g(s) g\left(s_{1}\right) \exp \left(i n \omega s+i l \omega s_{1}\right)
\end{aligned}
$$

The derivation this form of Eq.(A11) requires two important steps to be followed. The first step is just a Taylor expansion. While putting Eq.(A4) and (A5) in Eq.(3.36) and Eq.(3.37), we come across several integrals which contain one quantity in common in the integrands. This is an expression of the form $\exp \left[i k\left\{\phi(t)+\Delta \phi_{t}^{(1)}(s)\right\}\right]$, where $k$ is an integer. The first part, i.e. $\exp [i k \phi(t)]$, being a function of $t$, can be taken outside the integral while the second part can be dealt with as follows: 


$$
\begin{aligned}
\exp \left[i k\left\{\Delta \phi_{t}^{(1)}(s)\right\}\right] & =\exp \left[i k\left\{\Delta \phi_{t}^{(1)}(s)-\Delta \phi_{t}^{(0)}(s)\right\}\right] \exp \left[i k\left\{\Delta \phi_{t}^{(0)}(s)\right\}\right] \\
& =\left[1+i k\left\{\Delta \phi_{t}^{(1)}(s)-\omega s\right\}\right] \exp (i k \omega s)
\end{aligned}
$$

Here we have used Eq. $3.38 \mathrm{~b}$ ) and as an essential step (second) discarded the nonlinear terms. Also, since $\Delta J_{t}^{(1)}(\tau)$ and $\Delta \phi_{t}^{(1)}(\tau)$ are of order $O(\tau)$, we have

$$
\Delta J_{t}^{(1)}(s) \exp \left[i k \Delta \phi_{t}^{(1)}(s)\right] \simeq \Delta J_{t}^{(1)}(s) \exp (i k \omega s)
$$

Guided by the same physical reasoning, we assert that all integrals of the form $\int_{0}^{\tau} d s s F(s)$, with $F(s)$ being finite as $s \rightarrow 0$, yield terms of the order $\tau^{n}(n>1)$, and are hence neglected. In a similar way, we can obtain the second iteration on $\phi$. Thus,

$$
\begin{aligned}
\Delta \phi_{t}^{(2)}(\tau)=\Delta \phi_{t}^{(1)}(\tau)+\omega^{\prime} \sum_{n=-\infty}^{\infty} \sigma_{n} \exp (i n \phi) \int_{0}^{\tau} d s \int_{0}^{s} d s_{1} F\left(s_{1}\right) \exp \left(i n \omega s_{1}\right) \\
+\omega^{\prime} \sum_{n=-\infty}^{\infty} \sigma_{n} \exp (i n \phi) \int_{0}^{\tau} d s \int_{0}^{s} d s_{1} g\left(s_{1}\right) \exp \left(i n \omega s_{1}\right)+\sum_{n=-\infty}^{\infty} \sum_{m=-\infty}^{\infty} \sum_{l=-\infty}^{\infty}\left[C_{n m}^{\prime} \sigma_{l}-\right. \\
\left.i \quad(n+m) C_{n m} \mu_{l}\right] \exp [i(n+m+l) \phi] \int_{0}^{\tau} d s \int_{0}^{s} d s_{1} F\left(s_{1}\right) \exp \left[i(n+m) \omega s+i l \omega s_{1}\right] \\
+\sum_{n=-\infty}^{\infty} \sum_{m=-\infty}^{\infty} \sum_{l=-\infty}^{\infty}\left[C_{n m}^{\prime} \sigma_{l}-i(n+m) C_{n m} \mu_{l}\right] \exp [i(n+m+l) \phi] \int_{0}^{\tau} d s \int_{0}^{s} d s_{1} g\left(s_{1}\right) \\
\quad \times \exp \left[i(n+m) \omega s+i l \omega s_{1}\right] \\
-\sum_{n=-\infty}^{\infty} \sum_{l=-\infty}^{\infty}\left(\mu_{n}^{\prime} \sigma_{l}-i n \mu_{n} \mu_{l}\right) \exp [i(n+l) \phi] \int_{0}^{\tau} d s \int_{0}^{s} d s_{1} F(s) F\left(s_{1}\right) \exp \left(i n \omega s+i l \omega s_{1}\right) \\
-\sum_{n=-\infty}^{\infty} \sum_{l=-\infty}^{\infty}\left(\mu_{n}^{\prime} \sigma_{l}-i n \mu_{n} \mu_{l}\right) \exp [i(n+l) \phi] \int_{0}^{\tau} d s \int_{0}^{s} d s_{1} F(s) g\left(s_{1}\right) \exp \left(i n \omega s+i l \omega s_{1}\right) \\
-\sum_{n=-\infty}^{\infty} \sum_{l=-\infty}^{\infty}\left(\mu_{n}^{\prime} \sigma_{l}-i n \mu_{n} \mu_{l}\right) \exp [i(n+l) \phi] \int_{0}^{\tau} d s \int_{0}^{s} d s_{1} g(s) F\left(s_{1}\right) \exp \left(i n \omega s+i l \omega s_{1}\right) \\
-\sum_{n=-\infty}^{\infty} \sum_{l=-\infty}^{\infty}\left(\mu_{n}^{\prime} \sigma_{l}-i n \mu_{n} \mu_{l}\right) \exp [i(n+l) \phi] \int_{0}^{\tau} d s \int_{0}^{s} d s_{1} g(s) g\left(s_{1}\right) \exp \left(i n \omega s+i l \omega s_{1}\right)
\end{aligned}
$$

We next require to calculate the averages $\left\langle\Delta J_{t}^{(2)}(\tau)\right\rangle_{S}$ and $\left\langle\Delta \phi_{t}^{(2)}(\tau)\right\rangle_{S}$. By virtue of Eq. 2.10), the former yields,

$$
\begin{aligned}
& \left\langle\Delta J_{t}^{(2)}(\tau)\right\rangle_{S}=\left\langle\Delta J_{t}^{(1)}(\tau)\right\rangle_{S}-\sum_{n=-\infty}^{\infty} \sum_{m=-\infty}^{\infty} \sum_{l=-\infty}^{\infty}\left[B_{n m}^{\prime} \sigma_{l}-i(n+m) B_{n m} \mu_{l}\right] \exp [i(n+m+l) \phi] \\
& \quad \times \int_{0}^{\tau} d s \int_{0}^{s} d s_{1} g\left(s_{1}\right) \exp \left[i(n+m) \omega s+i l \omega s_{1}\right] \\
& \quad+\sum_{n=-\infty}^{\infty} \sum_{l=-\infty}^{\infty}\left(\sigma_{n}^{\prime} \sigma_{l}-i n \sigma_{n} \mu_{l}\right) \exp [i(n+l) \phi] \int_{0}^{\tau} d s \int_{0}^{s} d s_{1}\left\langle F(s) F\left(s_{1}\right)\right\rangle \exp \left(i n \omega s+i l \omega s_{1}\right) \\
& \quad+\sum_{n=-\infty}^{\infty} \sum_{l=-\infty}^{\infty}\left(\sigma_{n}^{\prime} \sigma_{l}-i n \sigma_{n} \mu_{l}\right) \exp [i(n+l) \phi] \int_{0}^{\tau} d s \int_{0}^{s} d s_{1} g(s) g\left(s_{1}\right) \exp \left(i n \omega s+i l \omega s_{1}\right)
\end{aligned}
$$

In Eq.(A15) we encounter three double integrals. The first and consequently the third are shown to be negligible in Appendix-B. For the second integral, viz. $\int_{0}^{\tau} d s \int_{0}^{s} d s_{1}\left\langle F(s) F\left(s_{1}\right)\right\rangle_{S} \exp \left(i n \omega s+i l \omega s_{1}\right)$, or $\int_{0}^{\tau} d s \int_{0}^{s} d s_{1} C(s-$ $\left.s_{1}\right) \exp \left(i n \omega s+i l \omega s_{1}\right)$, we refer to Ref. [10]. In Appendix-C of Ref. [10] this type of integral of more general form have been evaluated. Here we state the results only. 


$$
\begin{aligned}
I_{n, l}^{(j)} & =\int_{0}^{\tau} d s \int_{0}^{s} d s_{1} \int_{0}^{s_{1}} d s_{2} \ldots \int_{0}^{s_{j}-1} d s_{j} C\left(s-s_{j}\right) \exp \left[i n \omega s+i l \omega s_{j}\right] \\
& \simeq \frac{1}{(j-1) !}\left(\frac{i}{l}\right)^{(j-1)} \frac{d^{j-1} \tilde{\beta}_{l}(\omega)}{d \omega^{j-1}} \tau \delta_{n,-l}
\end{aligned}
$$

The important physical consideration here is to neglect the terms of order $\tilde{C}(\omega) / \omega$. The rest of the task amounts to solving the above integral, also known as the Dirichlet's condition for multiple integrals. Thus the integrals of the third term in the right hand side of Eq.(A15) reduce to,

$$
\int_{0}^{\tau} d s \int_{0}^{s} d s_{1} C\left(s-s_{1}\right) \exp \left(i n \omega s+i l \omega s_{1}\right)=\tau \tilde{\beta}_{l}(\omega) \delta_{n,-l}
$$

Putting Eq.A17) back into Eq.A15) yields, after a little algebra,

$$
\left\langle\Delta J_{t}^{(2)}(\tau)\right\rangle_{S}=\left\langle\Delta J_{t}^{(1)}(\tau)\right\rangle_{S}+2 \tau \sum_{n=1}^{\infty} n^{2} \frac{d\left|x_{n}\right|^{2}}{d J} \tilde{C}_{n}^{c}(\omega)
$$

which, after using Eq.(A8) reduces to,

$$
\left\langle\Delta J_{t}^{(2)}(\tau)\right\rangle_{S}=-2 \tau \sum_{n=1}^{\infty} n^{2}\left[\omega\left|x_{n}\right|^{2} \tilde{\beta}_{n}^{c}(\omega)-\frac{d\left|x_{n}\right|^{2}}{d J} \tilde{C}_{n}^{c}(\omega)\right]
$$

In a similar manner we also get,

$$
\left\langle\Delta \phi_{t}^{(2)}(\tau)\right\rangle_{S}=\omega \tau+\tau \sum_{n=1}^{\infty} n\left[\omega \tilde{\beta}_{n}^{s}-\tilde{C}_{n}^{s} \frac{d}{d J}\right] \frac{d\left|x_{n}\right|^{2}}{d J}-\tau f_{0}^{\prime} \mu_{0} t_{c}
$$

Next we step out for the final iteration stage, the third one.

\section{Third Iteration}

Inserting Eq.(A11) and Eq.(A14) in Eq.(3.36) and Eq.(3.37) as before we get the expansions for $\Delta J_{t}^{(3)}(\tau)$ and $\Delta \phi_{t}^{(3)}(\tau)$ which we write in the following convenient form.

$$
\begin{aligned}
\Delta J_{t}^{(3)}(\tau)= & \Delta J_{t}^{(2)}(\tau)-\sum_{n=-\infty}^{\infty} \sum_{m=-\infty}^{\infty} B_{n m}^{\prime} \exp [i(n+m) \phi] \int_{0}^{\tau} d s\left[\Delta J_{t}^{(2)}(s)-\Delta J_{t}^{(1)}(s)\right] \\
& \times \exp [i(n+m) \omega s] \\
& -\sum_{n=-\infty}^{\infty} \sum_{m=-\infty}^{\infty} i(n+m) B_{n m} \exp [i(n+m) \phi] \int_{0}^{\tau} d s\left[\Delta \phi_{t}^{(2)}(s)-\Delta \phi_{t}^{(1)}(s)\right] \\
& \times \exp [i(n+m) \omega s] \\
& +\sum_{n=-\infty}^{\infty} \sigma_{n}^{\prime} \exp (i n \phi) \int_{0}^{\tau} d s\left[\Delta J_{t}^{(2)}(s)-\Delta J_{t}^{(1)}(s)\right] F(s) \exp (i n \omega s) \\
& +\sum_{n=-\infty}^{\infty} i n \sigma_{n} \exp (i n \phi) \int_{0}^{\tau} d s\left[\Delta \phi_{t}^{(2)}(s)-\Delta \phi_{t}^{(1)}(s)\right] F(s) \exp [i n \omega s] \\
& +\sum_{n=-\infty}^{\infty} \sigma_{n}^{\prime} \exp (i n \phi) \int_{0}^{\tau} d s\left[\Delta J_{t}^{(2)}(s)-\Delta J_{t}^{(1)}(s)\right] g(s) \exp (i n \omega s) \\
& +\sum_{n=-\infty}^{\infty} i n \sigma_{n} \exp (i n \phi) \int_{0}^{\tau} d s\left[\Delta \phi_{t}^{(2)}(s)-\Delta \phi_{t}^{(1)}(s)\right] g(s) \exp [i n \omega s]
\end{aligned}
$$

and 


$$
\begin{aligned}
\Delta \phi_{t}^{(3)}(\tau)= & \Delta \phi_{t}^{(2)}(\tau)+\omega^{\prime} \int_{0}^{\tau} d s\left[\Delta J_{t}^{(2)}(s)-\Delta J_{t}^{(1)}(s)\right] \\
& +\sum_{n=-\infty}^{\infty} \sum_{m=-\infty}^{\infty} C_{n m}^{\prime} \exp [i(n+m) \phi] \int_{0}^{\tau} d s\left[\Delta J_{t}^{(2)}(s)-\Delta J_{t}^{(1)}(s)\right] \exp [i(n+m) \omega s] \\
& +\sum_{n=-\infty}^{\infty} \sum_{m=-\infty}^{\infty} i(n+m) C_{n m} \exp [i(n+m) \phi] \int_{0}^{\tau} d s\left[\Delta \phi_{t}^{(2)}(s)-\Delta \phi_{t}^{(1)}(s)\right] \\
& \times \exp [i(n+m) \omega s] \\
& -\sum_{n=-\infty}^{\infty} \mu_{n}^{\prime} \exp (i n \phi) \int_{0}^{\tau} d s\left[\Delta J_{t}^{(2)}(s)-\Delta J_{t}^{(1)}(s)\right] F(s) \exp (i n \omega s) \\
& -\sum_{n=-\infty}^{\infty} i n \mu_{n} \exp (i n \phi) \int_{0}^{\tau} d s\left[\Delta \phi_{t}^{(2)}(s)-\Delta \phi_{t}^{(1)}(s)\right] F(s) \exp (i n \omega s) \\
& -\sum_{n=-\infty}^{\infty} \mu_{n}^{\prime} \exp (i n \phi) \int_{0}^{\tau} d s\left[\Delta J_{t}^{(2)}(s)-\Delta J_{t}^{(1)}(s)\right] g(s) \exp (i n \omega s) \\
& +\sum_{n=-\infty}^{\infty} i n \mu_{n} \exp (i n \phi) \int_{0}^{\tau} d s\left[\Delta \phi_{t}^{(2)}(s)-\Delta \phi_{t}^{(1)}(s)\right] g(s) \exp (i n \omega s)
\end{aligned}
$$

It is needless to carry out further iterations because they yield terms of order $\tilde{C}(\omega) / \omega$ even in the order of $\tau$, and hence are negligible. From this point we therefore proceed to calculate the moments $\left\langle(\Delta J)^{m}(\Delta \phi)^{k}\right\rangle_{S}$ as the right hand side of Eq.3.25) demands. While performing the averaging, some integrals occur in common with both $\left\langle\Delta J_{t}^{(3)}(\tau)\right\rangle_{S}$ and $\left\langle\Delta \phi_{t}^{(3)}(\tau)\right\rangle_{S}$. They are as follows:-

$$
\begin{aligned}
& I_{1}=\int_{0}^{\tau} d s\left\langle\left[\Delta J_{t}^{(2)}(s)-\Delta J_{t}^{(1)}(s)\right]\right\rangle_{S} \\
& I_{2}=\int_{0}^{\tau} d s\left\langle\left[\Delta J_{t}^{(2)}(s)-\Delta J_{t}^{(1)}(s)\right]\right\rangle_{S} \exp [i(n+m) \omega s] \\
& I_{3}=\int_{0}^{\tau} d s\left\langle\left[\Delta \phi_{t}^{(2)}(s)-\Delta \phi_{t}^{(1)}(s)\right]\right\rangle_{S} \exp [i(n+m) \omega s] \\
& I_{4}=\int_{0}^{\tau} d s\left\langle\left[\Delta J_{t}^{(2)}(s)-\Delta J_{t}^{(1)}(s)\right] F(s)\right\rangle_{S} \exp (i n \omega s) \\
& I_{5}=\int_{0}^{\tau} d s\left\langle\left[\Delta \phi_{t}^{(2)}(s)-\Delta \phi_{t}^{(1)}(s)\right] F(s)\right\rangle_{S} \exp (i n \omega s) \\
& I_{6}=\int_{0}^{\tau} d s\left\langle\left[\Delta J_{t}^{(2)}(s)-\Delta J_{t}^{(1)}(s)\right]\right\rangle_{S} g(s) \exp (i n \omega s) \\
& I_{7}=\int_{0}^{\tau} d s\left\langle\left[\Delta \phi_{t}^{(2)}(s)-\Delta \phi_{t}^{(1)}(s)\right]\right\rangle_{S} g(s) \exp (i n \omega s)
\end{aligned}
$$

Integrals $I_{2}$ to $I_{7}$ are in common with $\left\langle\Delta J_{t}^{(3)}(\tau)\right\rangle_{S}$ and $\left\langle\Delta \phi_{t}^{(3)}(\tau)\right\rangle_{S}$ while $I_{1}$ occurs in the latter only. The integrands containing $F($.$) alone or in a product with g($.$) , on averaging gives zero by virtue of Eq.(2.10). And the integrands$ containing $g($.$) alone or two g$ 's in product, amounts to negligible contributions (see Appendix-B). The integrands containing the correlation average of two F's and not obeying (or can not be recast) the form of Eq. A16) have all been shown to be negligible by $\mathrm{CN}$ in their Appendix-C 10]. Such integrals as stated above constitute the bodies of $I_{1}$ to $I_{3}$. For $I_{4}$ through $I_{7}$, some integrals contain product of three terms viz. $\langle F(.) F(.) F(.)\rangle_{S},\langle F(.)\rangle_{S} g() g.($.$) ,$ $\langle F(.) F(.)\rangle_{S} g(),. g() g.() g.($.$) etc. The first two type of integrals vanish, the former due to Eq.(2.10) and the latter due$ to the Gaussian property of $F$. The last two types vanish, the latter following from Appendix-B and the former has been shown to be negligible in Appendix-C. The only non-zero contribution comes from $I_{5}$, the integral as a whole appears as $\omega^{\prime} \sum_{l=\infty}^{\infty} \sigma_{l} \exp (i l \phi) \int_{0}^{\tau} d s \int_{0}^{s} d s_{1} \int_{0}^{s_{1}} d s_{2} C\left(s-s_{2}\right) \exp \left(i n \omega s+i l \omega s_{2}\right)$, where we have used Eq. 3.5b). Using the result of Eq.A16 followed by a little bit of algebra we eventually arrive at the value of $I_{5}$. Thus,

$$
\begin{aligned}
& I_{5}=-\frac{i \tau \omega^{\prime}}{n} \sigma_{-n} \exp (-i n \phi) \frac{d \tilde{C}_{-n}(\omega)}{d \omega} \\
& \text { and } \quad I_{1}=I_{2}=I_{3}=I_{4}=I_{6}=I_{7}=0
\end{aligned}
$$


Thus we have,

$$
\left\langle\Delta J_{t}^{(3)}(\tau)\right\rangle_{S}=\left\langle\Delta J_{t}^{(2)}(\tau)\right\rangle_{S}+\sum_{n=-\infty}^{\infty} i n \sigma_{n} \exp (i n \phi)\left[I_{5}\right]
$$

which, by changing the dummy index from $-n$ to $n$ for this symmetric summation $(-\infty$ to $+\infty)$, and employing Eq.(3.12), 3.30) and (3.46g) and finally putting the value of $\left\langle\Delta J_{t}^{(2)}(\tau)\right\rangle_{S}$ from Eq. A18) we reach at,

$$
\left\langle\Delta J_{t}^{(3)}(\tau)\right\rangle_{S}=-2 \tau \sum_{n=1}^{\infty} n^{2}\left[\omega\left|x_{n}\right|^{2} \tilde{\beta}_{n}^{c}(\omega)-\frac{d}{d J}\left\{\left|x_{n}\right|^{2} \tilde{C}_{n}^{c}(\omega)\right\}\right]
$$

Similar calculations establish the result,

$$
\left\langle\Delta \phi_{t}^{(3)}(\tau)\right\rangle_{S}=\omega \tau+\tau \sum_{n=1}^{\infty} n\left[\omega \tilde{\beta}_{n}^{s} \frac{d\left|x_{n}\right|^{2}}{d J}-\frac{d}{d J}\left(\tilde{C}_{n}^{s} \frac{d\left|x_{n}\right|^{2}}{d J}\right)\right]-\tau f_{0}^{\prime} \mu_{0} t_{c}
$$

For the calculations of the second moments $\left\langle\left(\Delta J_{t}\right)^{2}\right\rangle_{S},\left\langle\left(\Delta \phi_{t}\right)^{2}\right\rangle_{S}$ and $\left\langle\Delta J_{t} \Delta \phi_{t}\right\rangle_{S}$ we refer the reader to the Appendices B and $\mathrm{C}$ of Ref. [10], where it has been clearly shown that terms which do not appear in $\Delta J_{t}^{(1)}(\tau)$ and $\Delta \phi_{t}^{(1)}(\tau)$ lead to cross terms or square terms containing three or more integrals over one or two $C$ functions. All of them contain higher powers of $\tau$ or terms of order $[\tilde{C}(\omega) / \omega]^{N}$ with $N \geq 1$, and are hence discarded. Thus we obtain the second moments as,

$$
\begin{aligned}
\left\langle\left(\Delta J_{t}\right)^{2}\right\rangle_{S} & =4 \tau \sum_{n=1}^{\infty} n^{2}\left|x_{n}\right|^{2} \tilde{C}_{n}^{c}(\omega) \\
\left\langle\Delta J_{t} \Delta \phi_{t}\right\rangle_{S} & =0 \\
\left\langle\left(\Delta \phi_{t}\right)^{2}\right\rangle_{S} & =4 \tau \sum_{n=1}^{\infty}\left|\frac{d x_{n}}{d J}\right|^{2} \tilde{C}_{n}^{c}(\omega)
\end{aligned}
$$

In obtaining the results of Eq. A28) to (A30) we have used Eq.A10a to Eq. A10d) and the like, along with the fact that higher order terms $\tau^{n}$ and $[\tilde{C}(\omega) / \omega]^{n}$ (with $n>1$ ) are negligibly small.

\section{APPENDIX B: EVALUATION OF INTEGRALS INVOLVING QUANTUM CORRECTIONS}

Here we calculate the terms involving single or multiple integrals of the quantum fluctuation $g($.$) keeping in mind$ the time scale of energy diffusion. First of all we recall the structure of $g(t)$ from Eq.(3.1f) and then refer to Eq.(A6). On the right hand side of that equation we have an expression of the form, $\sum_{n=-\infty}^{\infty} \sigma_{n} \exp (i n \phi) \int_{0}^{\tau} d s g(s) \exp (i n \omega s)$. For brevity, we set

$$
\frac{1}{(n-1) ! m !} \frac{\partial^{m}}{\partial t^{m}}\left[V^{n}(x(t))\left\{\left\langle\delta \hat{X}^{(n-1)}(t)\right\rangle-\left\langle\delta \hat{X}^{(n-1)}(0)\right\rangle\right\}\right]_{t=0}=\Upsilon_{m n}(0)
$$

so that, Eq.(3.1f) appears as

$$
g(t)=-\sum_{m=0}^{\infty} \sum_{n=3}^{\infty} t^{m} \Upsilon_{m n}(0)
$$

and we can write

$$
\begin{aligned}
\sum_{n=-\infty}^{\infty} \sigma_{n} \exp (\text { in } \phi) \int_{0}^{\tau} d s g(s) \exp (\text { in } \omega s) & =\sum_{n=-\infty}^{\infty} \sum_{l=3}^{\infty} \sigma_{n} \exp (\text { in } \phi) \\
& \times\left[\sum_{k=0}^{\infty} \Upsilon_{k l}(0) \int_{0}^{\tau} d s s^{k} \exp (\text { in } \omega s)\right]
\end{aligned}
$$

From Eq. (B1) it is obvious that 


$$
\Upsilon_{0 n}(0)=0
$$

and thus it follows that the right side of Eq. (B3) assumes the form $\sum_{n=-\infty}^{\infty} \sum_{l=3}^{\infty} \sigma_{n} \exp (i n \phi) \sum_{k=1}^{\infty} \Upsilon_{k l}(0) \int_{0}^{\tau} d s s^{k} \exp (i n \omega s)$ where the summation over the index $k$ now extends from one to infinity. Within the integral the exponential part fluctuates rapidly ( $\omega$ being very large ), remaining finite in the limit $s \rightarrow 0$. As it stands even for $k=2$ such integrals yield terms of order $\tau^{p}$ ( where $p>1$ ) [10]. Thus, integrals of the above form are negligibly small and are hence discarded. The case of $k=1$ calls for a special attention. The above expression takes a more simple form. Thus,

$$
\begin{aligned}
\sum_{n=-\infty}^{\infty} \sum_{l=3}^{\infty} \sigma_{n} \exp (i n \phi) \sum_{k=1}^{\infty} \Upsilon_{k l}(0) \int_{0}^{\tau} d s s^{k} \exp (i n \omega s) & \simeq \sum_{n=-\infty}^{\infty} \sum_{l=3}^{\infty} \sigma_{n} \exp (i n \phi) \Upsilon_{1 l}(0) \\
& \times \int_{0}^{\tau} d s s \exp (i n \omega s)
\end{aligned}
$$

In comparison to the rapidly varying exponential part, the linear part arising from the quantum fluctuation does not alter significantly within the range of integration and hence the right hand side of Eq.(B5) can be written as

$$
\begin{aligned}
\text { [right hand side of Eq.(B5)] } & =\sum_{n=-\infty}^{\infty} \sum_{l=3}^{\infty} \sigma_{n} \exp (i n \phi) \Upsilon_{1 l}(0) t_{c} \tau \delta_{n, 0} \\
& =\sum_{n=-\infty}^{\infty} \sum_{l=3}^{\infty}\left(i n x_{n}\right) \exp (i n \phi) \Upsilon_{1 l}(0) t_{c} \tau \delta_{n, 0} \quad \text { [using Eq.(3.30)] } \\
& =0
\end{aligned}
$$

Here $t_{c}$ is the cut-off time $(\simeq 1 / \omega)$ as has been mentioned earlier in the discussions preceding Eq.(3.48). Similarly, after averaging the last term of Eq. A5 becomes,

$$
\begin{aligned}
\text { [last term of Eq.(A5) }] & =\sum_{n=-\infty}^{\infty} \sum_{l=3}^{\infty} \mu_{n} \exp (i n \phi) \Upsilon_{1 l}(0) t_{c} \tau \delta_{n, 0} \\
& =-\mu_{0} t_{c} \tau f_{0}^{\prime}
\end{aligned}
$$

where we have used Eq.(3.31) and Eq.(3.46e).

Now consider the last integral of the right hand side of Eq. A15). It is of the form $\int_{0}^{\tau} d s \int_{0}^{s} d s_{1} g(s) g\left(s_{1}\right) \exp (i n \omega s+$ $\left.i l \omega s_{1}\right)$. Using Eq.(B2), it can be cast in the following form:-

$$
\begin{aligned}
& \int_{0}^{\tau} d s \int_{0}^{s} d s_{1} g(s) g\left(s_{1}\right) \exp \left(i n \omega s+i l \omega s_{1}\right) \\
= & \int_{0}^{\tau} d s g(s) \exp (i n \omega s) \int_{0}^{s} d s_{1} g\left(s_{1}\right) \exp \left(i l \omega s_{1}\right) \\
= & \int_{0}^{\tau} d s\left[-\sum_{m=0}^{\infty} \sum_{k=3}^{\infty} s^{m} \Upsilon_{m k}(0)\right] \exp (i n \omega s) \int_{0}^{s} d s_{1}\left[-\sum_{m_{1}=0}^{\infty} \sum_{k_{1}=3}^{\infty} s_{1}^{m_{1}} \Upsilon_{m_{1} k_{1}}(0)\right] \exp \left(i l \omega s_{1}\right) \\
= & \sum_{m=1}^{\infty} \sum_{k=3}^{\infty} \sum_{m_{1}=1}^{\infty} \sum_{k_{1}=3}^{\infty} \Upsilon_{1 k}(0) \Upsilon_{1 k_{1}}(0) \int_{0}^{\tau} d s s^{m} \exp (i n \omega s) \int_{0}^{s} d s_{1} s_{1}^{m_{1}} \exp \left(i l \omega s_{1}\right) \\
\simeq & \sum_{k=3}^{\infty} \sum_{k_{1}=3}^{\infty} \Upsilon_{1 k}(0) \Upsilon_{1 k_{1}}(0) \int_{0}^{\tau} d s s \exp (i n \omega s) \int_{0}^{s} d s_{1} s_{1} \exp \left(i l \omega s_{1}\right) \quad \text { [using Eq.(B5)] } \\
\simeq & \sum_{k=3}^{\infty} \sum_{k_{1}=3}^{\infty} \Upsilon_{1 k}(0) \Upsilon_{1 k_{1}}(0) \int_{0}^{\tau} d s s \exp (i n \omega s)\left[\frac{s \exp (i l \omega s)}{i l \omega}\right]
\end{aligned}
$$

where evaluating the third bracket of the last expression we have neglected terms of order $O\left(1 / \omega^{2}\right)$. The last expression yields terms of negligible contribution due to the reasons given at the onset of Eq. (B5). Integrals involving three $g$ functions can be similarly shown to be negligibly small. 


\section{APPENDIX C: TREATMENT OF INTEGRANDS OF $I_{4}$ TO $I_{7}$ OF APPENDIX-A}

In the discussions that appeared in between Eq. (A22g) and Eq. A23) we mentioned of four types of integrands which appear in the calculations involving the evaluations of the integrals $I_{4}$ to $I_{7}$ ( Eqs.(A22d)- (A22g) ). Let us recall their forms again. They are,

$$
\text { (1) }\langle F(.) F(.) F(.)\rangle_{S}, \quad(2)\langle F(.)\rangle_{S} g(.) g(.), \quad(3)\langle F(.) F(.)\rangle_{S} g(.), \quad(4) g(.) g(.) g(.)
$$

It was reasoned there that types (1), (2) and (4) yield terms of negligible contributions (see Appendix-B). Here we clarify the way of dealing with type (3). The specific forms of integrals involving this type of integrands are:-

$$
\begin{aligned}
& \text { (A) } \int_{0}^{\tau} d s \int_{0}^{s} d s_{1} \int_{0}^{s_{1}} d s_{2}\left\langle F(s) F\left(s_{1}\right)\right\rangle_{S} g\left(s_{2}\right) \exp \left[i \omega\left(j s+n s_{1}+l s_{2}\right)\right] \\
& \text { (B) } \int_{0}^{\tau} d s \int_{0}^{s} d s_{1} \int_{0}^{s_{1}} d s_{2}\left\langle F(s) F\left(s_{2}\right)\right\rangle_{S} g\left(s_{1}\right) \exp \left[i \omega\left(j s+n s_{1}+l s_{2}\right)\right] .
\end{aligned}
$$

These two integrals occur in $I_{4}$.

$$
\text { (C) } \int_{0}^{\tau} d s \int_{0}^{s} d s_{1} \int_{0}^{s_{1}} d s_{2}\left\langle F(s) F\left(s_{2}\right)\right\rangle_{S} g\left(s_{2}\right) \exp \left[i \omega\left(j s+n s_{1}+l s_{2}\right)\right] .
$$

This, with type $(A)$ above, occur in $I_{5}$. And lastly the integral,

$$
\text { (D) } \quad \int_{0}^{\tau} d s \int_{0}^{s} d s_{1} \int_{0}^{s_{1}} d s_{2}\left\langle F\left(s_{1}\right) F\left(s_{2}\right)\right\rangle_{S} g(s) \exp \left[i \omega\left(j s+n s_{1}+l s_{2}\right)\right]
$$

occurs in $I_{6}$ and $I_{7}$.

All the integrals from $(A)$ to $(D)$ lead to negligible contributions. We establish this by showing the case of, say, type $(B)$. For doing this we invoke the quantum fluctuation-dissipation relation from Eq.(3.5b) as,

$$
\begin{aligned}
\left\langle F(s) F\left(s_{2}\right)\right\rangle_{S} & =\frac{1}{2} \int_{0}^{\infty} d \omega^{\prime} \kappa\left(\omega^{\prime}\right) \rho\left(\omega^{\prime}\right) \hbar \omega^{\prime}\left[\operatorname{coth} \frac{\hbar \omega^{\prime}}{2 k_{B} T}\right] \cos \omega^{\prime}\left(s-s_{2}\right) \\
& =\int_{0}^{\infty} d \omega^{\prime} G\left(\omega^{\prime}\right) \cos \omega^{\prime}\left(s-s_{2}\right)
\end{aligned}
$$

with

$$
G\left(\omega^{\prime}\right)=\frac{1}{2} \kappa\left(\omega^{\prime}\right) \rho\left(\omega^{\prime}\right) \hbar \omega^{\prime}\left[\operatorname{coth} \frac{\hbar \omega^{\prime}}{2 k_{B} T}\right]
$$

where, a superscript “"” has been added as a superscript to $\omega$ to denote the frequency of the bath modes.

$$
\left\langle F(s) F\left(s_{2}\right)\right\rangle_{S}=\frac{1}{2} \int_{0}^{\infty} d \omega^{\prime} G\left(\omega^{\prime}\right)\left[\exp \left\{i \omega^{\prime}\left(s-s_{2}\right)\right\}+\exp \left\{-i \omega^{\prime}\left(s-s_{2}\right)\right\}\right]
$$

When Eq.(C1) is put in type $(B)$ integral along with Eq.(B2), we get the value of the integral as,

$$
\text { [type B] }=f_{0}^{\prime} \int_{0}^{\infty} d \omega^{\prime} G\left(\omega^{\prime}\right)\left[\frac{2 \omega^{\prime}}{n^{2} \omega^{2}-\omega^{\prime 2}}\right] t_{c} \tau \delta_{j+l,-n}
$$

This being of higher order in $1 / \omega$, can be discarded. It is easy to show that the other three types of integrals also lead to negligible contributions. The procedure is the same as that adopted above in the case of type $(B)$.

[1] H.A. Kramers, Physica (Amsterdam) 7, 284 (1940).

[2] E.W.-G. Diau, J.L. Herek, Z.H. Kim, and A.H. Zewail, Science 279, 847 (1998).

[3] A.W. Castelman, D.E. Folmer, and E.S. Wisniewski, Chem. Phys. Lett. 287, 1 (1998). 
[4] P. Hänggi, P. Talkner, and M. Borkovec, Rev. Mod. Phys. 62, 251 (1990), and the references given therein.

[5] V.I. Mel'nikov, Phys. Rep. 209, 1 (1991).

[6] Activated Barrier Crossing: Applications in Physics, Chemistry, and Biology, edited by G.R. Fleming and P. Hänggi (World Scientific, Singapore, 1993).

[7] New Trends in Kramers' Reaction Rate Theory, edited by P. Talkner and P. Hänggi (Kluwer, Dordrecht, 1995).

[8] R.F. Grote and J.T. Hynes, J. Chem. Phys. 73, 2715 (1980).

[9] P. Hänggi and F. Mojtabai, Phys. Rev. A 26, 1168 (1982).

[10] B. Carmeli and A. Nitzan, J. Chem. Phys. 79, 393 (1983).

[11] R. Graham and T. Tél, Phys. Rev. Lett. 52, 9 (1984); Phys. Rev. A 31, 1109 (1985).

[12] M. Arrayás, M.I. Dykman, R. Mannella, P.V.E. McClintock, and N.D. Stein, Phys. Rev. Lett. 84, 5470 (2000).

[13] B.C. Bag and D.S. Ray, Phys. Rev. E 61, 3223 (2000); 62, 4409 (2000).

[14] J.S. Langer, Ann. Phys. (N.Y.) 54, 258 (1969).

[15] A.M. Berezhkovskii, E. Pollak, and V.Yu. Zitserman, J. Chem. Phys. 97, 2422 (1992).

[16] C.R. Doering and J.C. Gadoua, Phys. Rev. Lett. 69, 2318 (1992).

[17] R.N. Mantegna and B. Spagnolo, Phys. Rev. Lett. 76, 563 (1996); V.N. Smelyanskiy, M.I. Dykman, and B. Golding, ibid. 82, 3193 (1999); J. Lehmann, P. Reimann, and P. Hänggi, ibid. 84, 1639 (2000); Phys. Rev. E 62, 6282 (2000); N. G. Stocks, Phys. Rev. Lett. 84, 2310 (2000); R.S. Maier and D.L. Stein, ibid. 86, 3942 (2001).

[18] A. Ajdari and J. Prost, C. R. Acad. Sci. Paris II 315, 1635 (1992); M.O. Magnasco, Phys. Rev. Lett. 71, 1477 (1993); R.D. Astumian and M. Bier, ibid. 72, 1766 (1994); R. Bartussek, P. Hänggi, and J.G. Kissner, Europhys. Lett. 28, 459 (1994); M.M. Millonas and M.I. Dykman, Phys. Lett. A 183, 65 (1994).

[19] J.M.R. Parrondo and P. Español, Am. J. Phys. 64, 1125 (1996); K. Sekimoto, J. Phys. Soc. Japan 66, 1234 (1997).

[20] R.D. Astumian, Science 276, 917 (1997); F. Jülicher, A. Ajdari, and J. Prost, Rev. Mod. Phys. 69, 1269 (1997); N. Thomas and R.A. Thornhill, J. Phys. D: Appl. Phys. 31, 253 (1998); P. Reimann, Phys. Rep. 361, 57 (2002).

[21] A.J. Leggett, S. Chakravarty, A.T. Dorsey, M.P.A. Fisher, A. Garg, and W. Zwerger, Rev. Mod. Phys. 59, 1 (1987).

[22] H. Grabert, P. Schramm, and G.L. Ingold, Phys. Rep. 168, 115 (1988).

[23] U. Weiss, Quantum Dissipative Systems (World Scientific, Singapore, 1993).

[24] J. Ray Chaudhuri, B.C. Bag, and D.S. Ray, J. Chem. Phys. 111, 10852 (1999).

[25] D.J. Tannor and D. Cohen, J. Chem. Phys. 100, 4932 (1994); D. Kohen and D.J. Tannor, ibid. 103, 6013 (1995).

[26] J.M. Sancho, A.H. Romero, and K. Lindenberg, J. Chem. Phys. 109, 9888 (1998); K. Lindenberg, A.H. Romero, and J.M. Sancho, Physica D 133, 348 (1999).

[27] R. Metzler and J. Klafter, Chem. Phys. Lett. 321, 238 (2000); Phys. Rep. 339, 1 (2000), and the references given therein.

[28] A.H. Romero, J.M. Sancho, and K. Lindenberg (unpublished), Preprint cond-mat/0204389.

[29] S.K. Banik, J. Ray Chaudhuri, and D.S. Ray, J. Chem. Phys. 112, 8330 (2000).

[30] J. Ray Chaudhuri, S.K. Banik, B.C. Bag, and D.S. Ray, Phys. Rev. E 63, 061111 (2001).

[31] D.J. Bicout, A.M. Berezhkovskii, A. Szabo, and G.H. Weiss, Phys. Rev. Lett. 83, 1279 (1999); D.J. Bicout, A.M. Berezhkovskii, and A. Szabo, J. Chem. Phys. 114, 2293 (2001).

[32] D. Banerjee, B.C. Bag, S.K. Banik, and D.S. Ray, Phys. Rev. E 65, 021109 (2002).

[33] E. Wigner, Phys. Rev. 40, 749 (1932); M. Hillery, R.F. O'Connell, M.O. Scully, and E.P. Wigner, Phys. Rep. 106, 121 (1984).

[34] A.O. Caldeira and A.J. Leggett, Phys. Rev. Lett. 46, 211 (1981); Ann. Phys. (N.Y.) 149, 374 (1983); Physica A 121, 587 (1983).

[35] P. Hänggi and U. Weiss, Phys. Rev. A 29, 2265 (1984).

[36] R.F. Grote and J.T. Hynes, J. Chem. Phys. 77, 3736 (1982).

[37] T. Hesselroth, Phys. Rev. E 48, 46 (1993).

[38] V.I. Mel'nikov, Zh. Eksp. Teor. Fiz. 87, 663 (1984) [Sov. Phys. JETP 60, 380 (1984)]; Physica A 130, 606 (1985).

[39] A.I. Larkin and Yu.N. Ovchinnikov, J. Stat. Phys. 41, 425 (1985).

[40] I. Rips and J. Jortner, Phys. Rev. B 34, 233 (1986).

[41] K.S. Chow and V. Ambegaokar, Phys. Rev. B 38, 11168 (1988).

[42] H. Dekker, Phys. Rev. A 38, 6351 (1988); H. Dekker and A. Maassen van den Brink, Phys. Rev. E 49, 2559 (1994).

[43] U. Griff, H. Grabert, P. Hänggi, and P.S. Riseborough, Phys. Rev. B 40, 7295 (1989).

[44] W.H. Louisell, Quantum Statistical Properties of Radiation (Wiley, New York, 1973)

[45] S.K. Banik, B.C. Bag, and D.S. Ray, Phys. Rev. E 65, 051106 (2002).

[46] H. Risken and K. Vogel, in Far From Equilibrium Phase Transition, edited by L. Garrido, Lecture Notes in Physics, Vol. 319 (Springer-Verlag, Berlin, 1988).

[47] M. Lax, Rev. Mod. Phys. 38, 541 (1966).

[48] M. Büttiker, E.P. Harris, and R. Landauer, Phys. Rev. B 28, 1268 (1983).

[49] H. Hippler, K. Luther, and J. Troe, Ber. Bunsenges. Phys. Chem. 77, 1104 (1973); K. Luther, J. Schroeder, J. Troe, and U. Unterberg, J. Phys. Chem. 84, 3072 (1980); B. Otto, J. Schroeder, and J. Troe, J. Chem. Phys. 81, 202 (1984).

[50] D.L. Hasha, T. Eguchi, and J. Jonas, J. Am. Chem. Soc. 104, 2290 (1982).

[51] J. Troe, J. Phys. Chem. 90, 357 (1986); J. Schroeder and J. Troe, Annu. Rev. Phys. Chem. 38, 163 (1987). 
[52] K. Hara, N. Ito, and O. Kajimoto, J. Chem. Phys. 110, 1662 (1999).

[53] A.N. Cleland, J.M. Martinis, and J. Clarke, Phys. Rev. B 37, 5950 (1988).

[54] R. Zwanzig, J. Stat. Phys. 9, 215 (1973).

[55] K. Lindenberg and B.J. West, The Nonequilibrium Statistical Mechanics of Open and Closed Systems (VCH, New York, 1990).

[56] J.K. Bhattacharjee, Statistical Physics: Equilibrium and Non-Equilibrium Aspects (Allied Publishers, New Delhi, 1997).

[57] A.K. Pattanayak and W.C. Schieve, Phys. Rev. E 50, 3601 (1994).

[58] B. Sundaram and P.W. Milonni, Phys. Rev. E 51, 1971 (1995).

[59] H. Risken, The Fokker-Planck Equation (Springer-Verlag, Berlin, 1989)

[60] R.P. Feynman and F.L. Vernon, Ann. Phys. (N.Y.) 24, 118 (1963).

[61] R.P. Feynman and A.R. Hibbs, Quantum Mechanics and Path Integrals (McGraw-Hill, New York, 1965).

[62] M. Topaler and N. Makri, J. Chem. Phys. 101, 7500 (1994), and the references given therein.

[63] J.-L. Liao and E. Pollak, J. Chem. Phys. 116, 2718 (2002).

[64] R. Creswick, Mod. Phys. Lett. B 9, 693 (1995). 